\title{
The Use of Molecularly Imprinted Polymers for Dermal Drug Delivery
}

\section{Suedee $\mathbf{R}^{*}$}

Molecular Recognition Materials Research Unit, Nanotec-PSU Center of Excellence on Drug Delivery System, Department of Pharmaceutical Chemistry, Faculty of Pharmaceutical Sciences, Prince of Songkla University, Hatyai, Songkhla 90112, Thailand

\begin{abstract}
Molecular imprinting has played an important role in the fabricating pre-defined drug selectivity in synthesized polymer products, which provides for significant changes in physicochemical property and recognition of its intended application. The physical features of imprinting the cross-linked materials generating a formed shape imprinted cavity have three-dimensional interaction sites contributing to chemical properties are useful for chiral separation of two isomers of asymmetric therapeutically agents through skin crossing biological barriers. The potential methods have created opportunities with advantages of tailor-made specific recognition sites for a given print molecule within an enantioselective molecularly imprinted membrane or nanostructure of engineered devices. Considering recent investigation regarding the in vivo behaviour of various formulation incorporated recognition system and release of desirable enantiomer of chiral therapeutic compounds, which demonstrated enhanced pharmacological effects of systemically administered pharmacologically active enantiomer of the chiral drugs as a result of use of delivery modalities. A number of thus the imprint formulations have been developed to provide for the diffusion of chiral specific molecules the challenges to realize the dream of producing effective biomimetic recognition systems. The review highlights fundamental aspects of chiral recognition processes of a given enantiomer as well as the production of binding site functionality still relies to a crucial extent on selective release of enantiomer of the racemic delivery, then indicate their current use in dermal drug delivery. This review discusses the possible future uses that they contribute advantageous drug delivery. In addition, the potential role of MIPs in studying the effect of physiological function as well as their specific mechanisms is given here.
\end{abstract}

Keywords: Molecular imprinting; Recognitive polymer; Barrier function; Biomembrane; Functionalized surfaces

\section{Introduction}

Manipulation of all knowledge emerging from various aspects of biomimetic recognition system has been a challenge for several applications including life science, bionanotechnology, pharmacy and medicine over the long term. A novel approach to design and creation of a recognition cavity is especially important, to implement the functionality of necessary stereochemical feature in artificial materials, and the discriminatory capacity of the specific sites for the pharmaceutically active agents. In particular, one of the most widely studied areas of advanced systems designed for molecular recognition is their utilization as a system that requires an application for enantiospecific analyses [1]. In this regard, recognition of the stereoselectivity and stereospecificty occurring in biologically relevant molecules of asymmetric compounds and biological process, the essential products of life are asymmetrical and such symmetry requires that any one stereoisomer is not superimposable on its image [2]. A means of incorporation of molecule-imprinted polymer material within a polymer is also providing opportunities of biomimetic recognitive system that a design framework-incorporated mirror-image form has been developed [3]. It is also worthwhile to look at constructing tailormade materials for stereospecific molecules [4]. An emerging technique of functionalized materials integrated with biocompatible biomaterials, based on premise of biochemistry and complementary chemistry that have been also performed for implication in the pharmaceutical sciences because the MIP-based devices should provide target delivery and safe passage for pharmaceuticals through the living systems. Drugs have pharmacological properties which they are given and adsorbed to the tissue site, providing their actions. The drugs have to be acted, biologically transformed and regulated by the biofunctional domains in the organized function of the body. In other words a smart strategy that served to fulfill a biomimetic interaction, whereby a polymer matrix was able to mimic specific recognition properties achieving in nature, yet present opportunity to create new materials highly tailored for the efficiently functioning and activity of a chiral material in the formed final product.

Enantiomers are molecular entities which exist as mirror image or handedness, with non-superimposible structures. Enantiomer has different arrangement around chiral center with the opposite form can have similar pharmacological activities but different potencies. In nonchiral environment enantiomorphs have the same physicochemical properties, and therefore cannot be distinguished under these conditions. However, transferring a pair of enantiomers from isotropic environment to asymmetric environment, two different diastereomeric species are formed with physicochemical features, in order to have all suitably enantioselective forces for rendering enantiomer separation in place. Biological elements as receptor or enzymes and whatever functional materials are capable of undergoing covalent and transient interactions with each of enantiomorphs, depending on the inherent nature of interactions between the chiral entity of compound and the stereochemical properties of recognition system. Discoveries showing that each enantiomorph of a chiral drug has different properties $[5,6]$. This indicates the important of stereochemistry on the administration

*Corresponding author: Suedee R, Molecular Recognition Materials Research Unit, Nanotec-PSU Center of Excellence on Drug Delivery System, Department of Pharmaceutical Chemistry, Faculty of Pharmaceutical Sciences, Prince of Songkla University, Hatyai, Songkhla 90112, Thailand, Tel: 66-74-288-862; Fax: 66-74- 428239; E-mail: roongnapa.s@psu.ac.th

Received May 24, 2013; Accepted August 12, 2013; Published August 24, 2013

Citation: Suedee R (2013) The Use of Molecularly Imprinted Polymers for Dermal Drug Delivery. Pharm Anal Acta 4: 264. doi:10.4172/2153-2435.1000264

Copyright: $\odot 2013$ Suedee R. This is an open-access article distributed under the terms of the Creative Commons Attribution License, which permits unrestricted use, distribution, and reproduction in any medium, provided the original author and source are credited. 
of stereoisomer of chirally active substances in mammalian body. These phenomena were occurred because of biological molecular recognition corresponds to optimal information content of receptor or antibody for a specific molecule. This dubbed double complementarity principle extending over energetical (electronic) as well as geometrical features. The geometrically well-defined cavities with spatial arrangement of atom, that is essential for a proper interaction with the site of action of the bioactive molecule. This allows for the stereospecificity of a series of chiral compounds to differ for different receptor systems and/or the enzymes involved, and thus are responsible for pharmacological activities.

The application of chemical principles to pharmaceutical systems that generally allows for administration of pharmaceutically racemate compounds when often one of them represents the more pharmacologically active isomer (eutomer), while the other one (distomer) might be even be active, but in a different way, and also produce side-effects, toxicity, or acts as an antagonist, is now being further developed. In other words, enantiomers have agonistic, antagonistic or synergistic properties in relation to its mirror image form. Nowadays, chiral separation techniques are becoming increasingly important in the pharmaceutical industry. The specific recognition ability to the molecule enantiomer via molecular imprinting polymers has become a significant demand for efficient processes in the field of analytical applications and drug development [7]. However, the development of such synthetic materials have been devoted to engineer via either conventional method or complex fabrication of stereospecific molecules within a rugged highly crosslinked polymeric network matrix with a high specificity for a given enantiomer molecule of a chirally active ingredient in a pharmaceutical product [8]. This will be beneficial not only lead to the feasibility of mimicking of molecular recognition of a desired enantiomer and then separation of stereoisomers, but which can also expedite the process of selectivity to the active site. In addition some of the drug delivery strategies that may overcome physical barrier of the skin which is of important by the implement in innovative methods of science, that they may have applications in dermal/transdermal drug delivery. Advances in the mechanistic understanding of the molecular level requirement that the inherent properties of a synthetic recognitive-based network are needed for the development of known therapeutic moieties. Whilst for artificial materials the receptor has to be created with a highly selective recognition. The process in fabrication of synthetic polymers with recognition has significance effect on chiral recognition capture to facilitate targeted drug delivery of chiral bioactive molecules or structurally closely related compounds through the barrier if the body prevents the molecule from getting to that site of action. As this review aims to provide a unique coverage of novel analytical method based on fabricating of synthetic polymers with high specific recognition against a chosen drug substance for recognition capabilities and delivery of pharmaceuticals, addressing the foundations of the enantioselective selectivity for helpful applications in research and drug development.

\section{Molecularly imprinted polymers (MIPs)}

The creation of new synthetic receptors have been increasing attention over two decades, more generally for its significance in intermolecular chemistry and in view of bioorganic implications. One may say that recognition of biological mimics of molecular imprinting like "lock and key". This significant supermolecule characterized by its thermodynamic and kinetic stability and the specificity can vary from one individual or part of an individual to another [9]. Among the different methods available for the polymer-based drug delivery system
[10], one of the most commonly used is the non-covalent imprinted polymers. In the non-covalent approach selective recognition materials can be achieved by equilibrating the enantiomer template molecules with an excess of suitable functional monomers in the presence of suitable cross-linking agents that can provide the required information to form a template with the correct size and shape and stereochemical features within their polymer systems that are fully complementary to a specific stereoisomer. Nevertheless production by the covalent bond method and cleavage requires tuning for the required performances. Molecular imprinting is an advantageous technique for producing highly cross-linked polymers with predetermined recognition sites for a specific chiral compound [11]. As a consequence biological recognition elements can be replaced by artificial materials but they are stable for long period of time, and even withstand under harsh conditions. This provides an alternative promising method for use to resolve a pair of enantiomers for chiral therapeutic compound containing pharmacological activities with residing side effect or toxicity in field of pharmaceutical research and development of drug candidates. One question raised is that how can a chiral compound possess essentially multiple blocking actions such that the eudismic ratio of different isomers or the macromolecule having different subgroups of specific functions, differs for different receptor types? To achieve highly selective recognition of the target species in the synthesized product it is desirable that shape binding site has substrate specificity. This is possible by making the drug of interest to be encapsulated with the designed engineering principles of polymers to establish numerous non-covalent binding interactions resulting in a tight-fitting unit and to sense its molecular size and contour of charge distribution towards original biological molecule. Therefore, the motifs of a molecular substance with high structure-specific recognition can be created within polymer matrix could then be tested either in test tube or in a living cell.

MIPs are highly cross-linked polymeric phases with pre-determined selectivity for a chiral molecule or a group of structurally related molecules [11]. Scheme 1 illustrates the basic principle of preparing for non-covalent molecular imprinting polymer. Selectivity needs to be introduced during preparation of the MIP with the solubility of a template molecule together with appropriate functional monomer in a solvent. A molecular assembly for template molecules, using building blocks of biocompatible networks and self-assembly is a very useful as it could be considered to be a process that mimics biological selfassembly with bioactive molecules. For self-organizing template and monomer into a molecular imprinting to yield a target-selective cavity

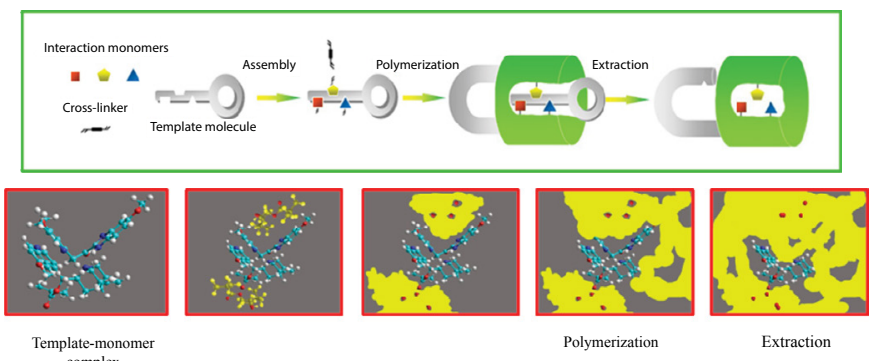

complex

Scheme 1: Illustration of the concept and typical generation of MIPs; they) produce polymer materials that are formed in the presence of a template molecule that can subsequently be removed and yet leave an imprint cavity in the polymer matrix that will recognize and allow for an environmentally sensitive reversible rebinding of a specific template molecule or structurally related compounds [7] 
template-monomer complex formation occurs, while the strength of which will depend on such factors as the degree of complementarity of the functional groups of the stereochemical template surrounding by the solvent molecules. After addition of crosslinking agent, whose hydrophilic or hydrophobic character can also be verified significantly, followed by polymerization (where the solvent act as a porogen and plays a pore-forming role). A washing scheme plays imperative role in the imprinting process, in addition the unreacted monomers, residues and template molecule are washed out from the network with a thermodynamically compatible solvent, yet providing for the diffusion and for accessible surface of specific binding site of the template molecule in the memory sites. The subsequent removal of the imprint molecules the binding sites that contain reversible intermolecular forces attach to entire pore-wall of imprints which the substrate molecule, can locate into, thus yielding suitable interactions can be rearranged with complexity of substance binding sites directed towards the target species. The molecular imprint of a racemate, isomer, or an isomeric mixture has the ability to transfer direct information on a sterically configured determinant for its biological activity to be transferred into a polymer material. As new technologies and biomedical applications, the design of stereochemically defined site of one-handedness related to MIP has to be formulated that allows for controlled release and efficient delivery at the drug target site. Although extreme molecular recognition may be achieved with rigidly, robust polymer by steric interactions, furthermore selective interaction requires a built-in flexibility inside well-defined nanosized environments, and yet a built-in flexibility which is of great importance in biological-receptor-substrate interactions that usually effect of interfacial interaction, adaptation and cooperative properties of both the species is needed to accumulation and organization i.e. bringing together binding sites and arranging them in a suitable pattern [12]. The key of pre-polymerization complexes, that allowed for selfassembly of the mixtures to form internal imprint cavities because of their non-covalent interactions with complementary functional groups inside the cavities involve to some synthetic effort and the choices of suitable material, and often it requires clearly defined optimization of binding agents. A strategic design can be served to meet the challenges of identifying recognition entities and specifically innovating in the required functionality, developing a diversity of different drug carriers and engineering delivery systems. The advances in polymer science and polymer templating technologies have opened new avenues in exploiting a wide variety of polymer and polymer systems for the field of pharmaceutical research and development of drug candidates that to fabricate the material sciences based on this new knowledge to enhance efficacy of drug therapy.

While molecular asymmetry usually has an effect on the number of interactions within a biological system such as a stereospecific therapeutic activity of a pharmaceutically related compound, or a chiral interconversion with the formation of a chiral metabolite. With respect to molecular asymmetry this usually has an effect on a number of its interactions within a biological system, such as stereospecific therapeutic activity of pharmaceutically related compounds or chiral interconversions and formation of chiral metabolites. Understanding of the significance of biological processes that depend on, these asymmetries; have provided the first insight into the stereospecificity of biological actions and the idea of receptors. The concept of molecular recognition occurs in biological system that the enzyme's target, or substrate, had to fit into a certain geometric space on the active binding site. This is a key issue for a chiral recognition process, via the features of either a single- or a multiple-point array that determined by the stereoselective interactions leading well stereospecific complexation of ligands with the spatially-oriented chiral sites. The generation of materials based on biomimetic imprinting the shape of active site of a natural enzyme or receptor in synthetic recognition materials, so that biological recognition elements can be replaced by artificial materials become advantageous in field of enantiomer separation and sensor for analytical application, such as biomimetic sensors, membrane separation [13] and in the fields of drug delivery systems [14]. This feasibility for developing a method for biomimetic moiety imprinting is mostly dictated by the molecular surface chemistry of interaction between the template and functional monomer or binding polymer chains. This chemistry can be modified by using tighter networks, increasing the amount of polymer used and using an appropriate chain length of cross-linkers all able to change the affinity, capacity, and release times [15]. However, the relative strength of non-covalent recognition interactions is really weak and intermolecular physical forces are essentially governed by the conditions of the medium and physiological parameters. The principles of the imprinted polymerization method depend on various intermolecular interactions between the functional monomer and the template while being surrounded by a cross-linking network. However there have been several reports that have garnered important structure-binding relationships between specificity and atomic composition, topography and shape of the template molecule. The rationally design MIP can be created to act as antibody biomimics, is directly consisted of component, which is of enormously important to contribute to our understanding of the biology and immunological aspects of the delivery of drugs. Thus the physical properties of molecularly enantioselective imprinted materials that play role to the substrates re-binding and de-binding to their imprinted sites such as the number of interactions between the functional monomer(s) and the template; the innate binding strength and specificity of the functional monomer(s) for the template and the nature of the noncovalent interactions employed, and from the mass transfer differences. The degree of their differences mostly depends on the specificity and stereoselectivity due to the size and shape of the template species. These include hydrophobic solvatophobic; electrostatic (ion-ion, ion-dipole, dipole-dipole); hydrogen bonding; dispersion and induction forces; $\pi-\pi$ stacking (face-face interaction); charge transfer interactions; the cooperativity of template substructures towards binding; the size of the template (sterics); the shape of the template; the spatial relationships between template substructures; the conformational flexibility of the template; hydrophobic fit with geometric match of hydrophobic areas; and multiple interaction/large contact areas. All of these will give some indication of the difficulty of forming a general set of rules for predicting the binding behavior of MIPs. When used in specific applications e.g. sensitive materials in pharmaceutical industry or for drug analysis which influence of MIP formulation on enantioselective release mechanism.

In contrast to natural products which display high levels of stereospecificity, synthesized compounds are often obtained as mixtures of stereoisomers such as racemates or diastereomers. (In some cases enantiomerically pure compounds are also driven via asymmetric synthetic protocols). These circumstances perhaps may change with the development of stereospecific catalytic methods, including biomimetic methods that might rely on the facilitation by enzymes acting as catalysts by employing biomimetic systems or enantioselective receptors. Stereoselective interactions of bioactive molecules with substrates produce consequences that trigger their different responses and pharmacological actions [16,17]. In addition various regulatory processes for exogenous chiral entities, especially 
pharmaceutically active compounds, with pharmacological activity, that produce unwanted side effects and toxicities because of the action of the distomer [18]. Consequence of the phenomena and enantioselective actions of chiral drugs administered to the body are considered important prototypes of the profiles of drug activities. In some situations, stereoisomers molecules with the same composition but differing in essential biological interactions are governed by the selective recognition of biological molecule, and therefore play important roles that differentiate the activities of pairs of enantiomorphs $[19,20]$. At the molecular level, pharmaceutical compounds or other bioactive molecules can be regarded as exogenous molecules that can selectively interact in stereospecific environments with particular enantiospecific sites of biological recognition system [21-23]. This, in turn, leads to detailed investigations of the pharmacokinetics, toxicological and metabolic activities of both stereoisomers that have become essential, providing range of therapeutically effect blood circulation and reduced toxicity. The creation of a critical idea is with one of these synthetic processes tempting a mimic of an original molecule since this strategy, based on the way the drug acts on by the body by using MIP as the binding agent allows it possible to enhance drug deposition and improve drug therapy. Such systems recognize such a particular molecule as biological systems making it possible to study the effects of drugs under physical condition or in the presence of metabolized enzyme or in an intact immune system providing development of diagnostic tests either in vitro or in vivo dominantly.

Molecular-specific recognition systems are extremely important issues that will require introduction of the effective synthetic protocols with regard to the tailor-made recognition polymers to be able to memorize the intended stereoisomer in an appropriate environment. For a MIP, they offer the possibility of providing for the direct production of a highly efficient and stereoselective matrix. In these systems possible advantages of enantioselective MIP materials are that they have potential advantages over immunoaffinity materials [24]. In proof-of-principle in utilizing this technique from the material of a recognition site, that relies on the influence of antigen-antibody interactions well established and is producing valuable consequence high (antibody-like) selectivity/affinity for templates and structurally closely related compounds and are stable with high physicochemical features, which would provide unlimited storage times, multiple-use and ease of preparation [25]. This is not quite similar to biological element, polymerization that the molecules while entrapped in an unchanged three-dimensional network of a polymerizing between functional monomer(s) and a cross-linking agent resulting in immobilized target species. MIP demonstrates great potential a recognition system owing to the affinity between the chemical entities of template, actually achieving generation of the binding site formation in molecularly imprinted polymers [26]. The latter aspect especially will require technical and intellectual support for the production of the MIP with enantioselective properties. Therefore, the development of a tailor-made interaction site of the resulting polymers form decipher a pharmacological and therapeutic point of view, as well as chirality issues, etc. which provides detailed structural properties as a 'mirror image' within a pore wall of the imprints. Strategies to produce improved well-defined non-covalent polymeric materials that will stabilize the respective assembled template-MIP complexes should provide an increase in the number of existing specific microbinding cavities. This allows for the advantageous and more interesting production of new synthetic recognition materials in biological sensors [11,12], artificial antibodies in immunoassay [25], synthetic receptor for affinity-based assays [27,28], and artificial enzyme systems for catalytic applications [29]. Perhaps the polymer-template self-assembly is a highly useful as an alternative attractive means for strategies of exploiting an oriented immobilization [30], especially facilitate insightful 'site-directed synthesis' experiments. A greater understanding in supra organization provides great opportunity for creating specific enantioselective recognition in the polymer material during the synthesizing polymerization and entire extraction the entrapped template and the recognition polymers exist the shape of originally surrounding framework remained. Thus the goals and strategies of a molecular imprinting technique link to the point of view of producing a shape that fits the molecule without affecting its activity inside a well-defined nanosized cavity in macromolecular crosslinked networks. This approach facilitates for investigation in biological recognition and applications in vivo for stereoisomers of medicines having different activities that can pave the way for new drug developments, screening candidates in discoveries of the drug and synthesis of lead compounds.

\section{The preparation process of enantioselective molecularly imprinted polymers}

In the last two decades several strategies in fabricating of molecular imprinting product have been exploited to generate selective recognition sites within a polymeric material. In any case, the tailored selectivity and precise control over the resolving process required for a rapid, inexpensive, and suitable recognition system that detailed knowledge of the mode of variation in the cross-linking network needed for a successful recognition. MIP can be designed to memorize a desired enantiomer structure, whereby both the stereochemical features and the corresponding surface chemistries with motifs of subsequently added initial pharmacologically important compounds are retained within the polymeric structure. However, the specificity and affinity of chiral entities of any current known polymer system is still perhaps not as high as those of occurring in natural enzyme and antibodies. A greater knowledge of host-guest interaction in an aqueous solution contributes to our understanding and solving some of seemingly insurmountable problems of biological barrier, and the scientific design for the implement of bioanalogous recognition abilities into a material to elucidate the forces that control guest interactions during complex biological processes. So, the judicious selection of a particular imprinting method is primarily determined by the inherent physicochemical properties and solubility of the template of interest. The choices of monomers and other ingredients such as the conditions under study are suitably varied corresponded to those for them in the intended applications [31]. Although there is a requirement for a novel imprinting method generating the specific imprinted cavities, that structure does not collapse upon the removal of the imprinting molecule. It is possible that an assembly of multiple weak interactions can produce a highly efficient binding for its efficient chiral recognition. Often, the conventional methods will provide the strategies or an innovative method generating creation of sensitive, selective synthetic polymers for a number of pharmaceutical compounds by molecular imprinting [32]. The interesting point about the enantioselective imprinted recognition system is the fact that both the stereochemical features and corresponding surface chemistries are retained in polymeric structure. By this approach can be utilized for producing an active chiral recognition site in their polymers by incubating a polymerization mixture containing the interactive functional monomer(s), which becomes a chiral moiety to determine the imprint (or the active chiral recognition sites)and that also accomplished by derivatization of a highly crosslinked polymer consisting of an achiral 
or chiral functional monomer. In a strongly competing surrounding environment, the functional imprinted materials with the site accessibility to analytes compromised, with unfavorable consequences with respect to chiral recognition capacity and selectivity. Nevertheless the alternative concept of producing polymer-embedded MIP cavities can be used by modulating the immediate selective environment in a favorable fashion (e.g. non-toxic and compatible phases) with a suitable assembly during the polymerization, yielding mimicking of three-dimensional structure to biological target [33].

\section{Alternative applications of biomimetic imprinting}

In nature, most biological processes are usually governed by mechanisms of molecular recognition. It is believed that in many cases the "receptor" was chiral and affinity of the enantiomers of the drug to receptor possessing its own pharmacological activity, side effect or toxicity. An explosion of novel synthetic drugs, were usually provided in their racemic form without regard to any potential problems. Figure 1 shows the chemical structures of some chiral drugs. These compounds thus can have vastly differing potencies and this is thought to be the way that the specific enantiomers of many important chiral drugs, such as chlorpheniramine, morphine, promethazine and propranolol, bind differently to their chiral receptor in the body with regard to the charge distribution at the interface of the interacting molecules and the spatial structural arrangement of the chirally imprinted structure. The process of clotting prevents excessive bleeding on injury in treatment for patients, whereas differences in the metabolism of warfarin; 7-hydroxylation is the most favored metabolic pathway for $(S)$-warfarin, whilst 6-hydroxylation is favoured by $(R)$-warfarin. In particular coumarins are competitive inhibitors of that process, and a number of them show stereoselectivity both in their therapeutic actions<smiles>CN(C)CCC(c1ccc(Cl)cc1)C1CCCCN1</smiles>

Chlorpheniramine<smiles>CC(CN1c2ccccc2Cc2ccccc21)N(C)C</smiles>

Promethazine<smiles>CC(=O)C[C@@H](c1ccccc1)c1c(O)c2ccccc2oc1=O</smiles>

(S)-Warfarin

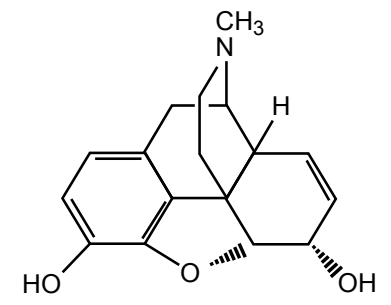

Morphine

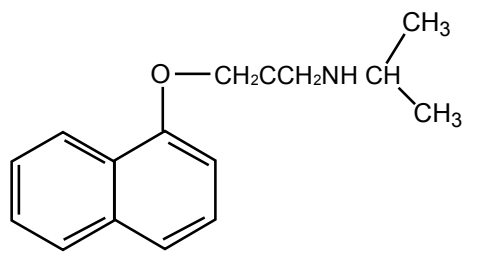

(R)-Propranolol

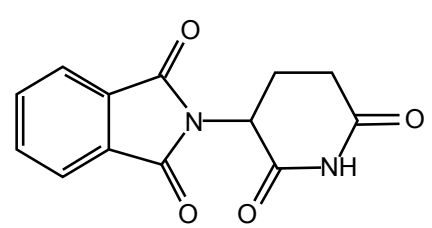

(R)-thalidomide
Figure 1: Chemical structure of some chiral drugs. and in their metabolism. The $(S)$-isomer of warfarin is several times more potent than its antipode as an anticoagulant [34]. Even though an increased awareness of potential problems the practice of administering racemates is expected to diminish so that the therapeutic activity of a single enantiomer and that the public concerns in adverse effect or toxicity of bioactive enantiomer forced both the scientific community and regulatory authorities to reconsider enantiomers. This will be particularly important for the next generation of highly potent drugs, such as peptides, genes and biomacromolecules, requires for analytical methods for quality control, which this raises an important problem and highlight separation of enantiomers from the racemic mixture. Most of the chiral imprinted matrixes incorporate separation phases in the sorption process that are target-specific with a reliable prediction of the separability of a given pair of enantiomers. The development of new enantioselective assay with use of enantioselective MIPs for a means of chiral resolution was established in a solid-phase extraction process [35], sensing systems and in drug bioanalysis for quality control and biomedical applications [36]. The chromatographic techniques in protein-bound and unbound forms of separation as specific probes has been reported, as to the examination of equilibrium binding constants, and allow average binding affinity of protein receptors that allows ready comparison of protein receptors of different sources [37]. It is one of the key challenges in rational design to implement of specific imprinted polymer for stereoselectivity of an enantiomer of interest. A strong potential benefit in the administration of chiral drugs will be achieved, since the central objective of a delivery system is to release therapeutics at the desired target site. Then, better therapeutic response of a biologically important, generating increase in the therapeutic window of racemic drugs. Therefore, the ability to regulate the rates of eutomer release from polymer or a dosage form consisting a racemate. In part because of the burdens associated with determining the profile and toxicities of mixed compounds, racemates have virtually disappeared from development as new molecular entities, composition profiling is an informative approach for investigating the effects of a chiral drug in pharmacological, toxicological well as clinical studies. As mechanisms of toxicity can be elucidated that would have become more apparent, impact of chirality on multiple aspects of toxicology. Therefore one enantiomer might be completely able to complex with a particular receptor or enzyme in a hydrophobic pocket and achieve appropriate orientation at a catalytic of appropriately aligning with either the active or the stabilization domain, yet rotational position. The method successfully pinholed compounds of different molecular entities but sharing a common target. This research showed that there were in fact differences in the underlying stereoselective interaction mechanisms of the racemic and enantiomers of an important pharmaceutically active compounds. Likewise, in case of MIP complex binding to surface with initial allergen proteins obtained from fresh rubber tree latex and manufactured gloves, the determination of the affinity distributions imprinted sites with specific recognition [38]. By sensor measurement, the determination of spatial distribution and arrangement of surface allergen imprinted layer affect protein attachment and subsequent protein binding can be achieved with cross-selectivity of binding affinity for related allergenic proteins on an inter-digitated electrode.

\section{Potential and benefits}

The scientific and economic relevance of producing designed recognition substances to use for biomedical purposes has in the last few years, spurred major developments in advanced drug delivery [39]. A number of the delivery system of drugs and consequently, the assumption has arisen that this will improve a drug's therapeutic action. Indeed, there is a growing awareness of these developments in 
the pharmaceutical industry. In this sense macromolecular polymers, for use as drug delivery systems, seem to provide a number of opportunities that require a detailed study. As a result of drugs becoming progressively more potent, it is essential to fully understand the role and importance of designing the drug delivery systems or carriers required for rationale drug therapy [40]. Of particular in chirally imprinted polymeric networks provide three-dimensional binding cavities that recognize a drug and maintain it at a therapeutic level both in the drug reservoir and at the target site, where it can be released to maintain an appropriate dose of drug over an appropriate time so that it is never present at a level that can cause side effects. However, there are still a number of obstacles with traditionally designed polymeric delivery systems that have limited their practical application. There has been a huge surge in the interest of using enantioselective MIPs as a means for improving the delivery profile of a variety of therapeutic active agents. This especially requires a well-defined molecular imprinted network, one able to bind and release only specific molecular species under a particular condition. In numerous cases, changes in environmental factors are too small to allow for any unambiguous mechanistic models to be established using experimental techniques. Further in vivo investigations are needed to confirm that such systems do create significant improvements over the previous well-defined drug delivery systems. Assessing the relative energetic contributions of individual sites and their abilities to release compounds (or even the process modes of recognition of the biological receptors with empirically established models) can be extremely difficult. An interdisciplinary of advanced material and physicochemical property of drugs in drug development has provided novel approaches for predominantly controlled release of drugs at the targeted regions for a prolonged period of time. The preparation of a standard component of such systems offers even more promising avenues to enhance good performance for intended application.

\section{Need and challenge}

Advances in the mechanistic understanding of the molecular level requirements of importance of the inherent properties of a synthetic recognitive-based network is creating challenges for the development of polymer recognition materials, parameter affects to function of the synthesized molecular imprinting polymers, potential for skin permeability enhancement by molecular imprinting techniques [32]. This is associated with the design of system that are capable of specifically interacting with the pharmaceuticals and formulation that can generate interaction sites with a greater sensitively for very low concentration of pharmaceuticals. Besides, the development of several imprinting technologies for achieving a suitable rate of diffusion and extend the level of therapeutics that there is still an urgent need to develop new ideas for fabricating the precursors of the imprinted materials that makes up the macromolecular networks. Different strategies can be utilized for producing an active chiral recognition site in a natural occurring substance and synthetic polymer [41]. The optimum environmental conditions are expected for the achieving of actual desired enantiomer recognition and subsequent selective desorption if very high selectivity can be attained, but they are needed to verify as a factor, which would enable modification of the recognition and enantioselectivity for template by one of the two stereoisomers of a racemic drug was used.

For the molecular optimal targeting by MIPs the enantiomer selection is of great importance due to optically pharmaceuticals undergoing racemization in vivo. It is intention to provide an entirely relation of the circumstance, by including established methods and advantages and limitation of general concepts for constructing complementary protocol of synthesis of a chiral MIP. Synthesized polymers with both the chiral molecular imprinted cavities for a given template in either chiral or non-chiral polymeric matrix have been used fabricating recognition system [42]. Also, research pointed out the importance of chemical engineering design of recognition system. Several influences on the physicochemical properties of re-uptake and release of the initial stereoisomer template in a polymeric environment are needed to overcome for limitations. Difficulties have been encountered by current carriers such as extravasation to specific drug targets. Other problems include: the stability of the drug carriers during storage and in vivo; drug loading; immunogenicity; degradability and release rate of drug. However advances in the field and establishment of viable strategic innovations may be devised to solve all these problems and achieve a standard goal in the field of biomedicine.

\section{Potential role of MIPs to dermal/transdermal delivery}

A combination of theoretical and experimental work would lead to an easier way of developing molecular recognition systems that they are comparable to their counterparts in the biological world. Recognizing the inherent complexity of biological systems, innovative strategies include designs for producing support materials and factors associated with a successful realization of a tailored recognition for advanced drug delivery across biological barriers [42]. This process could be greatly improved if there was an enhanced understanding of the underlying methodologies for producing engineered binding sites and for fabricating a special composition for advanced drug delivery across biological barriers. Strategies for producing an improved noncovalent imprinted material for chiral sites have been established by stabilizing assembled template-monomer complexes [43]. In concert with combinatorial chemistry may provide an opportunity for focusing chemical modifications of lead compounds. The use of molecular imprinting technique may be highly integrated in application of combinatorial chemistry to in vivo pharmacological and biopharmaceutical studies to optimize the in vivo properties of a drug candidate or may even be possible to design appropriate properties into therapeutic agents that are produced specifically for dermal or transdermal use [44].

Novel approaches and advanced drug delivery represents the ongoing research activities involve transport across biological barriers, which contribute an increasingly important problem for the drug development process. This issue is the result of interaction or reaction process of test compounds (e.g. new drugs, hit or candidates) to epithelial barrier (e.g. stratum corneum). This agrees in principle and would also involve to strategical role of in vitro barrier models in drug delivery research. The thematic issue fit well to development of new drugs. The considerable biological importance and biotechnology of biomolecule has attracted increasing interest in developing MIP artificial receptors capable of selectively binding biomolecules.

Strategies for the molecular imprinting approach have the potential advantage of use in such diverse fields as analytical chemistry, drug discovery and material sciences, each involving different steps in the MIP synthesis protocols. MIP may also be shaped in an appropriate format facilitates of interfacing enantioselective recognition development have been seen, exemplified by either a planar or vesicular layered nanoparticles. Attrition during the drug development process is a serious economic problem for the pharmaceutical industry and it is often a result of inappropriate physicochemical characteristics and related poor absorption and poor pharmacokinetics. The introduction of a combination of strategies has also proven to be advantageous for 
designing MIP systems in desired application [45]. In spite of many efforts that have been devoted to create specific cavities by means of template directed synthesis, the possibility of predicting ideal combination. The introduction of a combination of strategies has also proven to be advantageous for designing MIP systems for applications facilitates identifying biological entities in approach that arises from engineering devices coupled together to polymer with molecular recognition [46].

\section{Parameters for chiral recognition by MIP-based matrix}

Apart from the promising concepts of MIP-based device, considerable efforts have also concentrated on the formation of MIPs based on tailored interaction sites in polymeric material and biomimetic systems [11,47]. When designing or optimizing the enantioselective release using an MIP-based system, one focuses mainly on the following three criteria: resolution, control of release and the robustness of the designed systems [48]. Sufficient selectivity of the system and the absence of poor mass transfer characteristics are two requirements to meet this goal. The selective recognition of enantiomers by an imprinting polymer method is related to molecular recognition mechanism and the design of the polymer or its matrix for delivering a precise concentration of the drug or drug dose. For biomedical applications, a high enantioselectivity together with a high loading capability are essentially desirable attributes. In the following sections some of the various parameters employed for designing functionalities for MIP system or the development of MIP formulations, with an efficient recognition and a controlled release property of a given target species are briefly addressed.

In an analogous way to the binding of a drug to a receptor the spatial arrangement about a chiral center of a certain molecule can influence its ability to bind to a complex three-dimensional architecture that exhibits supramolecular chirality inside live tissues. Thus, the insights provided by these investigations into the phenomenon of molecular imprinting have established mechanism of chiral recognition by molecular imprinting and understanding for the extent of complex chemical formation in a polymeric environment from organized molecular entities into a chiral MIP [49]. Despite the systems and future premise of MIP in chemical recognition influences on the property of re-uptake and release of the initial stereoisomer template. Chiral recognition mechanisms by the imprinted cavities that influence interfacial interactions between the imprint and the enantiomer template needs for being addressed while presence of the function of the recognition site yet restoring the activity at the desired target site by triggered extra/intracellular environment. However, the importance of having binding groups of target or messenger molecule complementary to functionality of binding sites optimize the resultant chemistry based on what is considered to the efficient interaction between template and functional monomer within delivery device [49].

The ability to discriminate chirally at a specific site by an imprinted chiral agent is complementary principles concern the charge distribution at the interface of the interacting molecules and the spatial structural arrangement of the chirally imprinted structure [50]. However current findings indicate that the effect of manipulating structural parameters on the template binding parameters (such as affinity, capacity, selectivity, transport) is of tremendous importance in optimizing a formulation for a controlled release rate, rather than the loading capacity and mechanical strength in controlling key aspects of those phenomena [51]. In addition, the production of synthesized products that are molecularly imprinted polymer on their surfaces represents an alternative promising means that could be used for the controlled delivery of a drug also provides an opportunity to study membrane performance and the mechanistic recognition for drug therapeutics.

\section{The formation of MIP-template complexes}

The general features of the type of membrane or solid nanoparticle consisted of interface with MIP, additive, and the additional ingredients or a polymer to stabilize disposition and distribution, the surface and shell formulation partially resulted in complete release of drug through the whole imprints. An innovative method evolves to the development of advanced material to produce a reversible binding and desorption to the target species that are based on the formation of complexes between the preferred enantiomer and synthetic products. The primary basis of chiral recognition is the difference between the intermolecular interactions of the enantiomeric drugs, $A_{R}$ and $A_{S}$, and the binding agents, $M I P_{R}$ and $M I P_{S}$ which are the imprinted polymers for the recognition selectivity to particular optically pure compounds or at least they are enantiomerically enriched. The subscript symbol denotes isomer form of the chiral drug or analyte. These interactions are the cause for the following complexation equilibria which are described by the equilibrium constants $K_{R R}$ and $K_{S R}$, when assuming a simple 1:1 stoichiometry.

$$
\begin{array}{lc}
\mathrm{A}_{\mathrm{S}}+\mathrm{MIP}_{\mathrm{S}} & \mathrm{A}_{\mathrm{S}} \mathrm{MIP}_{\mathrm{S}} \\
\mathrm{A}_{\mathrm{R}}+\mathrm{MIP}_{\mathrm{S}} & \mathrm{A}_{\mathrm{R}} \mathrm{MIP}_{\mathrm{S}} \\
\mathrm{K}_{\mathrm{SS}}=\frac{\left[\mathrm{A}_{S} \mathrm{MIP}_{S}\right]}{\left[\mathrm{A}_{S}\right]\left[\mathrm{MIP} P_{S}\right]} & \mathrm{K}_{\mathrm{RS}}=\frac{\left[\mathrm{A}_{\mathrm{R}} \mathrm{MIP}_{\mathrm{S}}\right]}{\left[\mathrm{A}_{\mathrm{R}}\right]\left[\mathrm{MIP}_{\mathrm{S}}\right]}
\end{array}
$$

$\left[A_{S}\right],\left[A_{R}\right]$, and $\left[M I P_{S}\right]$ are the equilibrium concentrations of the free enantiomers and the polymer imprinted with a template of interest, respectively. The equilibrium constants, $K_{S S}, K_{R S}$ are related to the Gibbs Free Energies of the complexation reactions, $\Delta G_{S S}$ and $\Delta G_{R S}$, according to Equation (1).

$$
\Delta \Delta \mathrm{G}_{\mathrm{SS}}=-\mathrm{RT} \ln \mathrm{K}_{\mathrm{SS}} \text { and } \Delta \Delta \mathrm{G}_{\mathrm{RS}}=-\mathrm{RT} \ln \mathrm{K}_{\mathrm{RS}}
$$

In all instances where $\Delta G_{S S}$ is equal to $\Delta G_{R S}$ due to enthalpic and/or entropic effects in most instances both, the equilibrium constants for the formation of the diastereomeric complexes will be different. The ratio of the two complex constants can be defined as the thermodynamic (or intrinsic) complexation (or binding) selectivity of the enantiomer-MIP complex $(\alpha)$.

$$
\alpha_{\mathrm{SR}}=\frac{\mathrm{K}_{\mathrm{SS}}}{\mathrm{K}_{\mathrm{RS}}}
$$

The temperature dependence of the equilibrium constants can be approximately given by a simple form of the Van't Hoff equation, $\Delta H$ and $\Delta S$ being the enthalpy and entropy of complexation, respectively. (This approximation is possible only if no changes in $\Delta H$ and $\Delta S$ take place with $T$ and no changes in binding mechanism and/or in secondary equilibria occur with changes in $T$ ).

$$
\begin{aligned}
& \ln \mathrm{K}_{\mathrm{SS}}=-\Delta \Delta \mathrm{H}_{\mathrm{SS}} / \mathrm{RT}+\Delta \Delta \mathrm{S}_{\mathrm{SS}} / \mathrm{R} \\
& \ln \mathrm{K}_{\mathrm{RS}}=-\Delta \Delta \mathrm{H}_{\mathrm{RS}} / \mathrm{RT}+\Delta \Delta \mathrm{S}_{\mathrm{RS}} / \mathrm{R}
\end{aligned}
$$

The temperature dependence of the intrinsic selectivity is given by Equation (3).

$$
\ln \frac{\mathrm{K}_{\mathrm{SS}}}{\mathrm{K}_{\mathrm{RS}}}=-\Delta \Delta \mathrm{H}_{\mathrm{SR}} / \mathrm{RT}+\Delta \Delta \mathrm{S}_{\mathrm{SR}} / \mathrm{R}
$$

where $\Delta \Delta \mathrm{H}_{\mathrm{SR}}$ and $\Delta \Delta \mathrm{S}_{\mathrm{SR}}$ are the differences in the enthalpy and entropy 
of complexation between the enantiomers and are defined as the enthalpic and entropic energy of complexation between enantiomers and defined as $\Delta \Delta \mathrm{H}_{\mathrm{SR}}=\Delta \mathrm{H}_{\mathrm{SS}}-\Delta \mathrm{H}_{\mathrm{RS}}$, and in a similar way for the entropic term, which $R$ is the gas constant of $1.987 \mathrm{cal} \mathrm{mol}^{-1} \mathrm{~K}^{-1}$ and $T$ is the absolute temperature in Kelvin. The ratio of each binding constant obtained at different temperatures can lead to the total free energy, enthalpy and entropy. At a certain temperature, the enthalpic and the entropic contribution to the intrinsic selectivity cancel each other exactly. At this temperature, the complexation constants are equal for both enantiomers, the intrinsic selectivity coefficient becomes unity, although the template-MIP system is intrinsically potential enantioselective.

Two major sources are responsible for the different interfacial interactions between the template and functional monomers on the binding site within the imprinted cavity. Tighter binding (higher $K$ ) between one enantiomer and the functional monomer might be a source of reduced mass transfer and thus a slightly higher mobility of the complex. In this instance, and when using MIPs to assist with the drug transport, the drug diffusional selectivity counters the effect of the binding selectivity. Template-monomer complexes generally have slightly different $\mathrm{pK}$ values (This does not mean that these differences are large enough to allow for their resolution on the basis of this effect.) At $\mathrm{pH}$ values near to the $\mathrm{pK}$ values, even small differences might result in significant different net-charges for the complexes and thus in different interactions for the binding. The influence of binding capacity of MIP on the transport and selectivity of enantiomers. A set of at least 6-7 precisely determined data points per enantiomer is required for attaining reliable results for the two fitting parameters, $K$ and association capability, $\mu$. The number of MIP sites for which maximum selectivity, $\alpha$, is attained is commonly the optimum concentration of the MIP site. The higher the association constants, the lower is the amount of the MIP that corresponds to the maximum. In one example, this dependence of [MIP $]_{\text {opt }}$ on $K$ has been established [52]. In the case of predicting a shift of the selectivity maximum to a higher concentration of the selective site when the template-MIP complex was decreasing. This predicted the absence of a differentiable maximum value at finite concentrations when the effects of association of the template species or the bound MIP were zero or when the functional groups of imprinted sites were oppositely charged. This is also in agreement with temperature and the recognition ability of the enantioselective MIP-based system.

\section{Fundamental aspects in dermal drug delivery by enantioselective MIP-based matrix}

There have been increasing interests in biomimetic molecular imprinting as breakthrough technologies that the technique is straightforward, robust and that due to sufficient ruggedness to generate strong and highly selective systems possessing cooperative effects. In order to generate the sensitive materials with a threedimension ordered pore structure, for example, enantioselective MIP particles serve as a binding agent by being directly incorporated from a casting method [53]. On this way, the template enantiomer is allowed to attach to the existing MIP particle wherever there are void spaces on the particle surfaces that can be infiltrated with a casted solvent of a given polymer. Such a system shows an appreciable level of enantioselectivity with improved physical and chemical because of the intrinsic nature of the macromolecular chain differences and the high enantiomer separation characteristics. Tailoring this MIP format could provide a means for imprinting polymer particle surfaces with accessible binding site and introduce stereospecific binding cavities upon templating within both flat and sphere of solid matters.
An alternative advantage of the introducing a MIP system which allows stereoselective recognition of a drug, that would be enormously advantageous. The release of less pharmacologically active isomer or toxic isomer of a chiral drug is retarded, whilst, the release of desire isomer within a spatial well engineered device is selectively release to transport passing through any of biological membranes. Therefore, the ability to regulate the rates of eutomer release from polymer layer or a dosage form consisting a racemate, is of important and strong potential benefit in the administration of chiral drugs, where significant distinctly differential pharmacological activities and toxicity are important. In molecular imprinting an approach to surface immobilization of MIP materials allows to obtain thin layer of MIPs with binding sites located proximity to polymer surface [54]. The prepolymer mixtures are casted on solid support surface after polymerization and subsequent template removal, the resulting MIPs carrying surface binding sites. On the other hand, MIP thin-layers were synthesized at surfaces on the pores of membrane after derivatizing the support or underlying membranes with the polymerization initiator providing accessed site, as a consequence highly increase the efficacy of MIP. For this purpose, the polymer chains attached to the surface at the same time which helps facilitate the use of MIPs in advanced delivery of drug therapeutics, biopharmaceutical products and environmental substances. New approaches for application of MIPs offer even more promising avenues to improve drug therapy in future.

Another attractive feature of functionalized materials can achieve both an efficient chiral separation and enantioselectivity towards a template isomer. Thus, if for a given enantiomer a molecular surface imprinted approach has been focused on tailoring an MIP format within a polymeric environment using biocompatible macromolecules, to allow for an improved fitting to the template and the selective construction of a selective shape while still providing an ability to protect the binding groups of a chiral therapeutic molecule from the potentially deleterious effects of moving solvent molecules. This of course would not apply in those cases that are involved in the re-binding of template. Some types of activity are dependent on a flat structure, while other types of activity require a bulky threedimensional molecule. The MIP cavities formed are in size, shape, and functional-group arrangements complementary to the templating molecule, and therefore can act as a target-specific binding site. Factors that can help to create complementary shaped cavities with a greater selectivity are the underlying chemical and physical properties which relate to the molecular parameters such as dielectric constant, dipole, polarizability, and hydrogen bonding capability of the solvent in which they are dissolved that the solvent should not interfere features of the template with the polymer matrix. An aqueous solvent may compete with crucial template-functional monomer interactions and thus any intermolecular hydrogen bonds and dipole-dipole stacking motifs exposed to the solvent are readily disrupted under polar solvent conditions and thus the efficacy of the imprinted binding sites. On the other hand, the size and shape of a therapeutic active molecule can be a significant feature in its biological action.

A very promising research utilizing methods that involve a number of different techniques to prepare monoliths, spherical beads and composite built from polymer system or coated polymer achieving either via the useful tools of direct synthesis or in situ polymerization and even surface grafting polymerization process for achieving of imprint surface selectivity [55]. Moreover, utilizing the surface-immobilized binding site imprinted with the enantiomer printed molecule and then incorporated in the membrane have been recently developed. Whereas the synthetic membranes obtained from 
Citation: Suedee R (2013) The Use of Molecularly Imprinted Polymers for Dermal Drug Delivery. Pharm Anal Acta 4: 264. doi:10.4172/21532435.1000264

Page 9 of 23

the biomaterials and self-assembly of the complementary functional groups by polymerizing monomeric components containing a given template into a confined entity as a result of direct the generation of organized functional structures in equilibrium conditions [56]. The presence of an immobilized molecular imprinted polymer surface with an ability to selectively delivery a particular specific molecule. In this respect the diversity of these synthetic polymer particles and that may include some that have molecular coupling capabilities to immobilize proteins, or polymers that change shape on heating or reversibly soluble polymers. On the other word, the diversity of the synthetic polymers used may include polymers that have molecular binding capabilities to immobilize proteins, or some polymers that change shape on heating or reversibly soluble polymers. With a multifunctional monomer anchoring to the binding site within an imprinted cavity, the interacting parts of both the MIP and a template undergo specific structural rearrangements that is of important which lead to the observed tight complex. Figure 2 illustrates the preparation process and particular features of various formats of MIPs when incorporating such molecular recognition surfaces into the nano/microparticles that

Imprinted granules

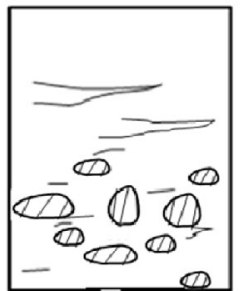

Bulked polymer is crushed ground and sieved to an irregularly defined sized particles

MIP microsphere

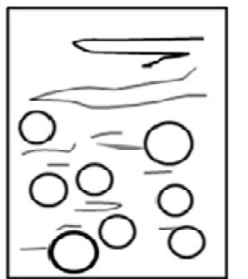

Emulsification of monomeric mixture and pre-

polymerization using a

rotating blade at $250 \mathrm{rpm}$

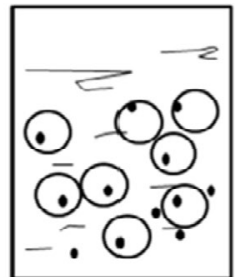

Add drug microsphere form

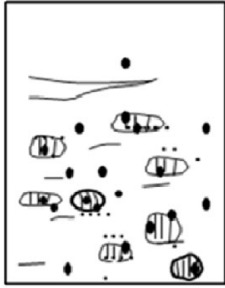

Add drug and ground bulked powder

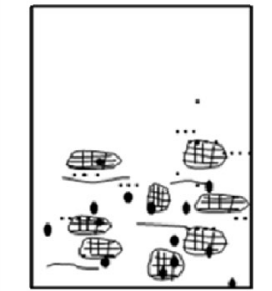

Drug adsorbed onto the surface of the polymer

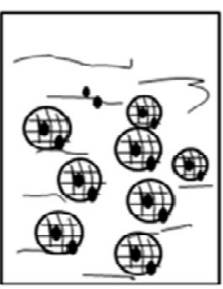

Microparticulate MIPs incorporated drug

\section{为 \\ Separate the particles from unincorporated drug by sedimentation}

\section{Microspherical MIP nanostructure}

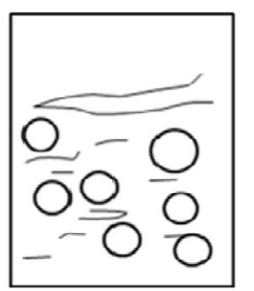

Emulsification of monomeric mixture in the presence of template

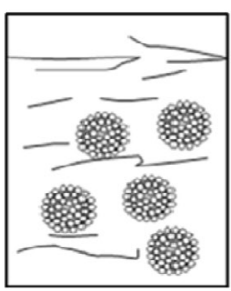

Cross-linking and polymerization under vigorous stirring using a controlled magnetic stir bar

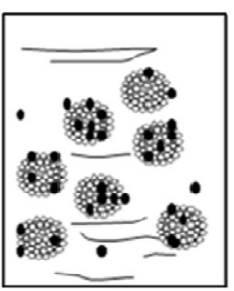

Add drug onto the MIP microsphere with nanostructure
Imprinted nanostructure

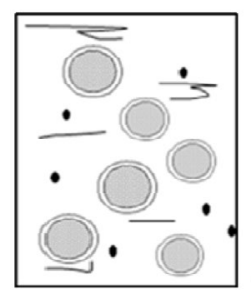

Double polymerization of the layer and compenent onto the surface of incorporated drug on imprinted beads

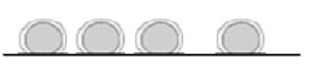

Removable of MIP microshere from non-incorporated drug by filtration and washing

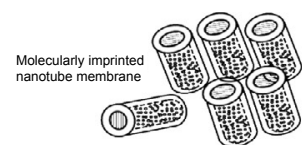

Figure 2: Schematic illustrations of the preparation process and particular features of various formats of MIPs for enantioselective-controlled drug delivery [4,55,56]. 
represent an attractive alternative approach, which turned out the facility of enantioselective controlled drug delivery.

One format of the MIP requires that is the presence of an immobilized molecular imprinted polymer surface with possibility of accessible site and an ability to selectively deliver a particular specific molecule upon desorption process of the target analyte in complex matrix. In this respect the diversity of these synthetic polymers may include some that have molecular coupling capabilities to immobilize proteins (e.g. antibodies), or polymers that change shape on heating or reversibly soluble polymers. A network which is imprinted for a particular drug can exhibit an improved drug release profile, by providing controlled release of the therapeutic moiety and an extended residency for the unwanted enantiomer, and can also be used to increase drug loading within the network, or to select a specific enantiomer with a higher therapeutic value. With a multifunctional monomer anchoring to the binding site within an imprinted cavity, the interacting parts of both the MIP and a template undergo specific structural rearrangements, that lead to the observed tight complex [57]. The high stability of the MIP-enantiomer complex can be seen as the end point of a process of mutual fitting, rather than as the result of a built-in shape that is ever coincidently complementarity between the interacting partners, which turns out to be very advantageous in chiral recognition process for biomedical applications. Consequently molecular organizations in a given drug molecular are crucial for understanding pharmacological and toxicity mechanisms in biological systems. Either forms of organization in biomimetic system performed by preparing selectively permeable enantioselective membranes using a molecular imprinting technique present a way to understand the molecular organization of a drug used clinically.

Minimizing the nonspecific adsorption of target compound or bioactive molecule is important in order to preserve the performance of the membrane. Hence, modification strategies for the control of a selective release should include ways to produce biocompatible membranes. One of the prominent of molecular imprinting method is that creating molecular recognition in material, where combined approach to the use of a material can selectively recognize and respond to variations in the analyte concentrations and trigger a controlled level of active determined by this molecular recognition event. And, because of the ensuring simplification of the MIP matrix, this has the possible potential for incorporation of a triggered-drug delivery system allow for extracellularly activated release or as an interesting tool to trigger transformation since in nature receptors at cell membranes can recognize specific external signals and initiate an appropriate cellular response. The mechanisms, of the extent and consequences of receptor co-localization and inter-receptor communication may significantly enhanced the understanding how a protein receptor and enzyme activity in their skin and elicit luminescence or fluorescence [58], as illustrated in Figure 3a. On the other hand, the real benefit of attachment of sensing technology additionally, the presence of the fluorescent MIPs having a fluorophore stemming in the imprinted cavities make them amenable to mechanistic scrutiny by fluorescence spectroscopic techniques could be visualize through skin. The derived mechanistic rationales helped to establish method optimization parameters by a genuine pattern of discrete fluorescence and evaluation of the binding kinetics with sample surfaces exposing molecularimprinted polymer. When considering the differential activities of a pair of drug enantiomers, that requires the isomeric print molecule to be involved in the preassembly process of the monomers with the binding group congeners involved in chiral recognition followed by a polymerization into a polymeric chain network that possesses surfaces (a)

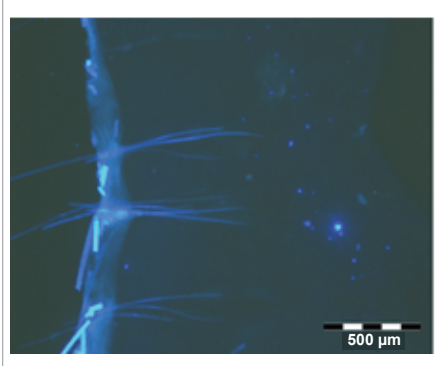

(b)

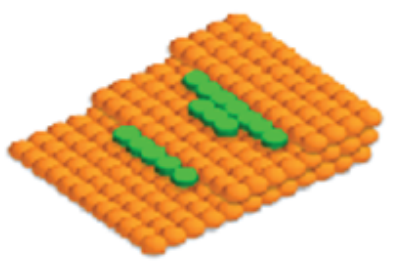

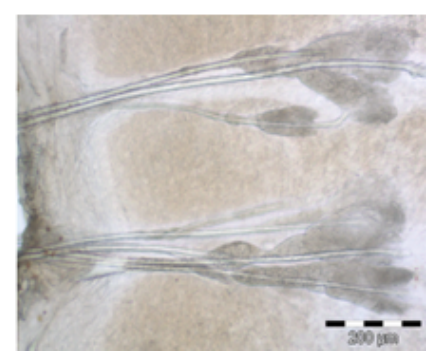

(c)

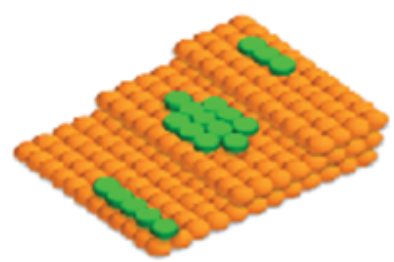

(d)

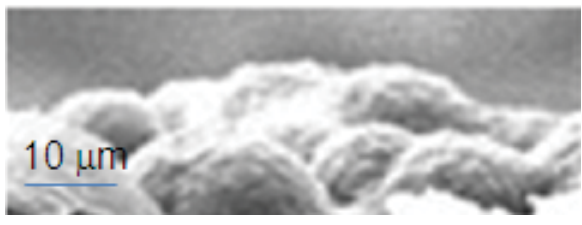

(e)

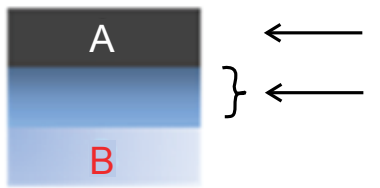

Surface-imprint

Interfacial diffusionzone

(f)

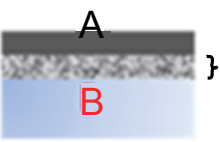

New compound reactive boundary
Figure 3: Top: illustration of topography and scanning electron microscope of microspherical MIP. (a) Left: fluorescent image of for rat skin dorsal represent image of cell receptors and enzyme activity led to fluorescence can be detected by fluorescent microscopy method, right: compared to the bright field image of rat skin [58]. (b) the relative rate of diffusion, accommodation and deposition determine the interfacial interaction and morphology on the barrier kinetic balance of a surface process where facile transport of the delivered substance no interlayer transport occurs and diffusion is rapid before attach of molecules and materials can occur in starking contrast (c) step down diffusion the intermolecular forces between the delivered species is rapid with high interlayer transport. (d) the hierarchically ordered surfaces forming between MIP nanostructures and cast membrane by self-assembly polymerization, showing ordered solid/liquid coexistence. Bottom: Two possible interfaces that can be formed between a membrane consisted of MIP materials (e.g thin-layer or microparticle) onto the skin: (e) non-abrupt interface and (f) reactive interface [69].

and confined accessible binding sites that the degree of stereoselectivity demonstrated was considerably higher [32]. Therefore, strategies for the preparation of membrane adsorbers could be applied also to 
membranes used for dialysis and for cell and tissue culture. An example of an even more advanced biomaterial is membranes prepared for the culture of adherent cells. This function is based on the specific capturing of a cell surface target by changing the conformation of a grafted copolymer prepared into a $\mathrm{pH}$ - or temperature-gating membrane. This range of biological behavior is marked the method based on antibodyantigen interactions, represents an alternative promising approach for drug carrier or almost any other in vitro bioassays allowing for the development of responsive, activated surfaces with a very specific response that mimics natural responsiveness. The key question that has fascinated biomedical scientists has been how to design and control material properties to achieve a specific biological response.

\section{The scientific considerations for process of MIP in dermal drug delivery}

The transfer of a drug from an aqueous topical solution across the outermost layers of the epidermis depends upon the ability of the molecule to diffuse through the vehicle, and stratum corneum and across the interfaces between the stratum corneum and vehicle and viable tissue. Hence, the drug-release profiles depend on various physicochemical parameters: vehicle-membrane partition coefficient for the drug, the ratio of drug-diffusion in the vehicle to the membrane and the ratio of drug-diffusion in the vehicle to membrane thickness. Enantioselective imprinted polymers consist of macromolecular materials and their preparation depends on generating an accurate molecular template for the enantiomer of interest for absorption of the drug passing the intercellular domains of the stratum corneum, which lipid-rich component, and then the epidermis permeability barrier occurs. MIPs as enantioselective materials have properties that can be used with high recognition capability which is of great important in enantiomer separation science. Despite the wide application of a chiral MIP in composite and carrying membranes for chiral separation and drug bioanalysis, their use, particularly in the delivery field falls into the modulated drug release based on: stereoselective recognition; release of particular structures from racemic mixtures of pharmaceutically active compounds; making artificial implants biocompatible and non-toxic; allowing drug release (due to membrane dissolution) only when particular environmental conditions occur; and simulating the permeation properties of natural tissues. For these purposes, it is taken from the two important formats categories: externally regulated and self-regulated systems, both rely on the recognition property and penetration permeability by the imprinted cavities. With growing the nano level to the meso level and the macro level with internal structural organization for MIP nanostructure incorporating within support membrane, template binding to imprinted sites can either change the pore network, therefore altering membrane permeability. In general the permeation rate is controlled by the interaction with the well-defined binding sites within micropore walls, thus functional integration and external connection, or even self-connected. For example, the modifications could activate a combination of several functions, via the creation of the surface such as on surface of the polysaccharide membranes with biomimetic recognition structures [59]. For tailoring and optimizing MIP matrix/membrane of its recognition function, it is critically important to control the affinity of MIP sites along with their density in the membrane and to create a well-defined pore and intended membrane morphology.

The choices of polymer or systems depend on their physicochemical properties that can produce efficient MIP and allow for the release of the delivered molecules through a relatively free permeation. The appropriate materials should be a perfect host for the incorporating procedure in terms of compatibility, and the components of the resultant MIP material should have no negative effects on the properties of the templated molecule because of any hostile conditions or mechanical stresses during the templating process upon interfacial process [60]. In addition, the components of the mixture should be retained by the molecular imprinting system to enable a biotherapeutic activity of any pharmaceutical compounds in response to some particular signal that depended on the design of the system used for incorporating the therapeutic agent. Obviously, the choice of the MIP will depend on various other factors such as the degree of membrane swelling, biodiversity, biocompatibility, and interactions with the drug and excipients or components of polymers. When the MIP is embedded in the polymeric matrix, membrane or any dosage formulation it is important to preserve its intrinsic feature of the imprinted cavities and to allow the diffusion of the solvent into the polymeric environment to the location of selective sites. Consequently, the template enantiomer that interacts strongly with the MIP will diffuse first, in advance of the less strongly interacting enantiomer. The MIP recognition materials are still capable of selectively binding to the enantiomer of a certain molecule that is structurally similar to the print molecules, and therefore may give rise to differential rebinding in the imprinted pores and desorption of the template binding [61]. There is requirement for a certain thickness for optimizing the process and for precluding the possibility of some mismatching of the membrane perturbation energy that will not affect the functionalization of the selective materials, for example the nanotube MIP nanostructure and within internal pores. In a subsequent release study using these membranes it was found that it had enantioselective transport properties with the template permeating more rapidly, with excellent enantioselectivity in vivo [62]. This approach to imprinting represents an attractive alternative method for the chiral separation of structurally related analogs with similar stereochemicals to the enantiomeric template from a racemic formulation. This necessitates the presence of a chiral environment, required to render enantiomers distinguishable. The introduction of a drug to a MIP may lead to enhanced efficacy for the drug by differential distribution of the desired isomer of chirally pharmaceutical active compound in body tissues before the release of the active form. Decreased tissue distribution restricts the action of a drug to a specific target site in the body and so decreases the toxic side localization of drugs has been achieved with use of a non-toxic synthetic material with predefined recognition which release the active drug within the target site. Drug design based on molecular imprinting approach can, of course, only be applied to existing drugs or basic types associated with a known action but it means that once a desired biological activity is detected in a parent molecule the pathway of the development to therapeutically acceptable product is more direct and sure than previously. Based on the principle of opposite enantiomer mobilities when applying surface-immobilized MIP it was possible to efficiently separate a pair of enantiomers of a chiral drug by the specific selective sites with the recognition characteristics after administration of the drug enantiomers into blood stream, by oral route in the animal model [62].

On the other hand, dermal route of administration has the advantage. Such a strategy represents an alternative means of transdermal drug delivery when a highly metabolized drug is protected from first-pass elimination. In addition, the short plasma half-lives of the steady state conditions to be achieved rapidly. MIPs are capable of selectively-controlled delivering drug stereosiomers and that there certain parallels between their in vitro results and the efficacy of the pharmacological active isomer in animal model. The cumulative 
amount of drug that diffuses through the stratum corneum into the epidermis at any specific time is of interest when analyzing transport properties. In addition the release rate of a drug from its release formulation is necessarily controlled by the overall concentration of the drug at a level that allows for both pharmacological activity and safety.

The critical drug activity at the receptor site is usually related to blood and other distribution fluid levels, as well as elimination rates. Other factors affecting activity include deposition sites, biotransformation processes protein binding and the rate of appearance in the blood. Hence in order to obtain the desired response, the drug must be absorbed both in sufficient quantity and at a sufficient rate. On the other hand, dermal route of administration has the advantage. Various aspects of the metabolism of pharmaceuticals and xenobiotics in the skin have been studied, and it has been shown that skin contains various enzymes including esterases which are capable of hydrolyzing different esters [63]. In certain cases this selectivity can be attributed solely to solubility differences between pure enantiomers and racemates. Moreover, formulation aims in the light of bioavailability considerations, are to produce a drug delivery system such that doses contain the intended quantity of drug and the drug is usually released but always in a controlled manner, in order to achieve the required onset, or if a long duration of response, sustained release is considered. Modification in structure of substance might produce an active compound suitable for skin transport. The approach represented a crucial key essential for the non-covalent bindings, that led to the cooperative effects that resulted in the structural diversity that was being directed by inter and intramolecular interactions that implemented the molecular recognition process in the complex matrix inside the biological barriers [64].

Once carrier-mediated epithelial transport processes are involved. As may be derived from the available kinetic data, directed transporters appear to exhibit high substrate selectivity as well as stereoselectivity. When a drug racemate is administered, the two enantiomers may be in similarity to their interaction with receptors exhibit similar or different affinities to the respective binding sites. When both enantiomers are transported via the same macromolecule, they may compete at the binding site. In addition, the biological barriers may affect the rate and extent of drug input into the systemic circulation, some with the potential for steroselectivity, due to the involvement of chiral macromolecules, and some without this potential. Thus if the eutomer of chiral drugs and analogues are to be selectively transported across the skin a better therapeutic response might be expected relative to that obtained using a racemic mixture of the drug. The rationale for designing the binding agents which enable stereoselective and trans-membrane transport of the drug depends on two fundamental assumptions: (a) the therapeutic effect of its administration arises from an increase in the plasma concentration in body compartments and (b) this increase is fostered by the desired enantiomer found in plasma that can diffuse into fluid from racemic compounds from the molecularly imprinted polymer.

\section{Formulation strategies to overcome skin barriers}

Percutaneous drug delivery is the transport of drugs from a formulation, through the stratum corneum into the viable epidermis followed by distribution into the blood capillaries and the lymphatic system. The advantages of transdermal drug delivery over the other routes of administration have been well documented, including avoidance of variable absorption rates and initial metabolism with oral delivery and improved therapeutic activity and bioavailability. Control/ tuning of surface chemistry and interfacial properties, enable assembly, allow bottom-up self-assembled surface chemistries and provide guided patterning of biological molecules for fundamental research or practical integration in functional polymer. Challenge of trying and integrating the data of drug delivery upon administration in biological system to key to decide what it means and determine what to do next. The results of a screening run tell us something about supra molecular complementarity between the binding species, which in turn tells us something about changes at target site. Despite the physicochemical properties of the membranes: solubility, hydrophobicity, and localized charge along the lipophilic domains that can be partially controlled by changes in biological conditions.

The protective layer of stratum corneum can be modulated in number of ways. In some cases, however, the outer most layer of skin, the stratum corneum, serves as the actual barrier against water loss from the body an also penetration of most the foreign and exogenous agents. Subcutaneous is in principle a two-compartment-system. It consists of elongated keratinized corneocytes embedded in extracellular highly ordered liquid bilayers responsible for the essential function. In addition the drug delivery that may overcome biophysical nature of the skin that they may have application in dermal/transdermal drug delivery. For example tested, it certainly cannot follow through all the other metabolic processes that are essential to replace in the enzyme in vivo. A difference in the observed enantiomeric ratio for the template with some cross-selectivity, that can provide a diversity of conformational structure and stereochemical properties relative to the template isomer $[56,61]$. On the other hand among the many ingoing avenues for this approach turn out to be beneficial due to a reduction in the metabolism of the delivery species and the therapeutic agent can cross the stratum corneum by using molecular imprinting. The use of polymer mimicking with chiral recognition the ability of the cavities imprinted with the enantiomer template pose complementary hydrogen bonding to the functionalities of MIP binding sites. In this regard, physical size of the imprinting isomers must fit better to enter the recognition site rather than complementary preferential conformation of enzymes resulting in catalytic and activity in loss-balance of physicochemical properties than the opposite enantiomer under biological microenvironment in the epidermis and the disappearance of stereospecificity of the enzyme's behavior by metabolic reaction it catalyzes eventually. Otherwise if this chain of insertion for the polymer crosslink for recognition is broken the species cannot thrive and multiply. All of these potential complex interactions between the biophysical barriers and delivered enantiomer therapeutics, in which penetration with the release from binding imprints and remaining excess within the entrapped matrix the MIP system offer the opportunities for enhancement of stereoselective transport and serve to protect the stereochemical structures of more pharmacologically active isomer (eutomer) conferring a useful shelflife to chiral pharmaceutical active compounds. In simple in vitro biophysical testing techniques when the applying to skin as a drug delivery route, assessing the fate of delivered compounds need for the accessibility, often relating to biomembrane research. Moreover, in vitro models for the evaluation of absorption and configurational conversion are definitely required in the development of optically active compounds for drug delivery. It is dramatically shown that the dependence of the effective selectivity on the MIP is of major importance to be considered for enantioselective controlled release of specific substance [65]. A much enhanced diffusion rate of the enantiomer templates or structurally close related molecules to the skin surface that was comparable to the diffusion through the stratum corneum, due 
to the molecule spending more time as the result of a lower diffusion coefficient [66]. With a large diffusion coefficient the mobility of molecules for the enantioselective imprints, produce an imbalance of the enantiomers in the aqueous donor vehicles. The features responsible for these proven stereospecificities of the enantioselective imprinted cavities can affect differentially absorption and diffusion to produce a workable system and goes some way towards validating such MIPs as potential receptor mimics. Otherwise the incorporation of special MIP within the biocompatible biomaterials influences synergistic effects and the mixture of enantiomer are retained by the MIP based matrix but indicated that interaction was sensitive to small structure differences in configurational of three-dimensional arrangement in skin [66]. The permeability that it is sensitive to conformation in a complicated but informative way and spectral changes. Furthermore, a release study is conducted the constituents that influence the binding strength, even if they do not affect the intrinsic selectivity of the system, can affect the effective selectivity at a given concentration of MIP. This has been demonstrated for the influence of organic solvent components and for the buffer concentrations and ionic strength. The different methods for effective enantiomeric separation can be achieved from two (independent) stereoselective phenomena. The first arises from the differences in the capacity and strength of the interactions between the enantiomers and their binding to the MIP material.

With regard to possibilities of modulating the penetration of drugs into skin, the study in systemically the influence of promising fatty acids on either change the drug escaping tendency (thermodynamic activity) due to changes in the solubility of the drug in its vehicle or the barrier resistance of the subcutaneous. Although membranes need to be passed during the absorption process and membranes are composed of chiral elements, it is generally assumed that stereoselectivity is not an issue with drugs that are passively absorbed by lipid diffusion, whereas difference between enantiomers are feasible when active transport mechanisms are involved. However in order to appreciate their potential role the physiological barrier of skin relevant to drug delivery are given. Thus, design and strategy of more selective features of MIPs are used to indicate interactions in addition to configurational change at asymmetric centre atom and enriched enantiomer and feasible Saffman and Delbrück mobility of the transferred species, especially cross physiological barriers to get to their sites of action. For the primary route of exposure in skin drug delivery, the organization of the intercellular lipid domains is considered to be important that can form into small macroscopic domains necessarily to biophysic feature of the stratum corneum. The drug delivery of hydrophobic drugs from topical formulation across skin partition and diffuse through the hydrophilic viable epidermis and dermis before uptake by the cutaneous microcirculation. However, the layer with barrier existed either to large molecules or nanoparticles. The polymer mosaics will not only bind the community together, but will also form compartments, of differing hydrophobicity and diffusivity throughout which the pharmaceutical substances must interact. However, the layer provides a barrier to the diffusions of micro/nanoparticles. It is possible to employ the self-organized structures molecular imprinting to pharmaceutical advantage in the transdermal drug delivery of enantiomers of chiral compounds. The complexation in saturated solutions involved to the diluting of the drug in similar vehicle and using MIP system which the degree of saturation reflect the skin penetration of the pharmaceutical, according to the recognition ability. While removing of the enantiomer probes from the skin surface the source of inward and outward fluxes that serve as the reservoir, so that the imprinted polymer incorporating in a membrane support can control the rate of enantiomer release.
Because the resistance to the transdermal flux of charged drugs can be reduced by disruption of the stratum corneum, by modification or by increasing the lipophilicity of the ionized permeant. Furthermore, interfacial of both attached layer and surface tension as well as the bending rigidity are of particular important factors on the transport of the drug loaded MIP materials when place on the stratum corneum and then change of the vesicle and that for the lateral organization can be similar, kinetics play an important role in determining the final configuration [67]. With molecular imprinting explanation in surface free energy of skin into the relative rate of diffusion, accommodation and deposition determine the interfacial interaction and morphology on the barrier kinetic balance of a surface process. On the basis of in vitro strict isomorphous replacement, thermodynamically and kinetically differences of species which appear to be feasible excludes from binding in endogenous receptors or metabolic enzymes. The whole process of imbibing an element, or energy-rich compound, of it traversing many membranes, of its concentration being buffered at each stage and of it being excreted involves in many complexing reactions. First, transport of the substance no interlayer transport occurs and diffusion is rapid before attach of molecules and materials can occur, whilst an another type of process, step down diffusion the intermolecular forces between the delivered species is rapid with high interlayer transport as illustrated in Figure $3 \mathrm{~b}$ and $3 \mathrm{c}$, respectively. The higher interlayer transport high amount of deposition, the greater number of the adsorbed layer of contact.

Skin penetration studies have usually focused on the physicochemical factors affecting the transport of drugs across the skin. The change in drug's physicochemical properties might produce an active compound suitable for skin transport, although the drug after absorption through the stratum corneum is subsequently metabolized to the parent compound. There is also some evidence for enantioselective differences in transport across skin [68]. Despite routes of permeation have been debated for many years and the precise mechanism of transfer across the stratum corneum has not yet been unequivocally determined. This is strong evidence that suggests that the intercellular route is important the intercellular regions of the stratum corneum comprise an expanded, structurally complex of which play important role in percutaneous transport. Other ways of delivering the sensitive drugs as skin as the delivery route for systemic effects are therefore required. The routes by which this may be accomplished, which involves drug administered via stratum corneum barrier in pharmacologically active stereoisomer. As illustrated in Figure 3d and 3f, two possible interfaces for the delivery devices and the barrier layer interfacing: with interfacial diffusion zone or providing reactive boundary. For non-abrupt interface, advanced modifications could activate a combination of several functions, via the creation of biomimetic recognition structures on the membrane surface: shielding, in order to avoid the adsorption and denaturation of proteins via hydrophobic or ionic interactions, selective adsorption and stabilization of the conformation of adsorbed proteins, covalent immobilization of biomolecules, or induction of biomimetic effects via synthetic structures. Secondly, reactive interface which represents the specific capturing or the controlled release of substances with the binding agent under an external environment, that is increasingly integrated into biomedical applications. Shape selective pores have been generated surface immobilization of the MIP materials (e.g. nanoparticles, membrane) which involves change in conformation or configurational modifications within a hybrid material or polymer system [69].

Candidate physiologies include dormant quiescent cells, metabolic enzymes, and expression of efflux pumps, as well stereoselective 
penetration of particular compounds [70]. The functional barriers of these corneum adsorptions appear to depend on their linkages between genes to create an interpretation of the genetic interaction networks that may lead to new avenues for therapeutic intervention. The alternative advantage of these biological mimicking polymers provides insights into understanding the biofunctional and self-organization within living tissues that will be valuable for clinical implications. There has been a plethora of experiments to determine percutaneous absorption in vitro and it is important to select a design where the transport is limited by the skin and not in any stagnant diffusion layer placed to the underlying skin surface, and which this is related to bio membrane research [71,72]. The applicability of composite, functionalized MIP has shown to be advantageous to the elucidation of the normal functioning of the skin when the template enantiomer molecule binds to the respective MIP, the active triggers the scintillator of living cells to generate a response signal $[7,73]$. In this way the imprinted polymer can be looked as an alternative means - it directly induces facilitation in the molecular event of target binding and balance obtained from the limitations of barrier and diffusion but other than the results lead to be efficient target transfer with high selectivity - enantiomer transport to improve the bioavailability across the membrane depend greatly upon the nature of the drug. Clearly, the binding capacity of the polymer with the ability to get drug through barrier, the level of agent used therapeutically, the distribution of mass and local hydrodynamics, together with the rate of turnover of the template-MIP binding relative to drug enantiomer diffusion rate [74]. The volume and relative capacity of a membrane support would be insufficient to deplete the bulk availability of the enantiomers, and chiral specific-target sites within the matrix polymers would become saturated with adsorbed/reacted drug enantiomers. The net effect once again would be to delay, rather than to prevent, in the physical function of epidermis (e.g. rat skin), and that they may have application in transdermal drug delivery which is also discussed as below.

By utilizing a biomimetic recognitive system of a self-assembly polymerization or polymer system-incorporated mirror-image that allow for the controlled release and selective delivery of specific substance, then improve bioavailability of small organic drug molecules with rapid passage across biological barrier into the blood circulation [75]. For such purpose, the polymerization between cross-linking agent and a polymerizable monomers with template present that exhibited an accommodating environment for molecularly imprinted sites in the polymer which is included in the underlying base that both static and dynamic processes made possible for the selective and reversible re-inclusion into the original surrounding polymeric macromolecular [76]. Aggregation creates regions of reduced chain mobility in the manner of polymeric crosslinks with adaptation providing functional groups loosely attached to those of template complementary in the surrounded by crosslinking structure. When the amount of the templating species increased in deeper layer clusters of the aggregates cause regions of the polymer to behave differently, and some at which higher the imprint site continues to increase, the regions of restricted motion begin to overlap and a single. The optimal placement of binding sites of imprinted polymers should be near the cavities. In the event that the interaction between a template and MIP is being fabricated with a favorable manner which deposition of the system has been reduced to a thin layer of soft tissue interposed between the polymer surfaces [77].

Some of the strategies for fabricating the delivery devices together with selective materials that actually facilitate, the molecule's ability to cross the physiological barriers which the key is their ability to recognize specific molecules [78]. In addition the specific binding of a particular enantiomer imprinted polymer will improve its chemical stability, and even prevent inversion or hydrolysis degradation of enantiomer during physical process and administration of the drug. To transfer the enantiomer, the use of a particular solvent has an impact not only on the enantioselective controlled drug delivery of the resulting polymeric material across biological barrier; it also is an essential component inherently involved in the enantioselective interaction between the template and functional monomer at multiple levels. The components of the solvent medium - bulk solvent, buffers and additives - not only dictate the conformational preferences of a flexible template and their functional monomers; their functional groups are also capable of complementary intermolecular interactions. The adsorbed solvent may give the mobility necessary to change the morphology in a decorated pore which the swelling of the films made of hydrophilic and hydrophobic components of the copolymer results in the reorganization and extend for random coil conformation [79].

Advances in the area of skin permeation together with information of the use of molecular imprinting approach provide as a result of research in chemical defense and efforts to identify the reasons for the barrier properties of the skin and how these properties could be further modified and use in the system and prediction. Considering to the surface free energy, of skin or change in the Gibbs energy, where the surface tension components of liquid that is unfavorable for addition of matter to small clusters up to a critical size. After attaining the critical nucleus size, further addition of material to the binding site is energetically favorable. The formulation of this size depends on the change in Gibbs energy [80]. The change in surface free energy of skin for forming a different spherical MIP of radius $r$ from number of $N$ is

$$
\Delta \mathrm{G}=-\mathrm{N} \Delta \mu+4 \pi \mathrm{r}^{2} \gamma
$$

The first term is the chemical potential change brought about by the phase transformation of $N$ atoms into a sphere with a surface energy given by the second term. The surface term is surface area times the surface tension, $\gamma$ of the bulk interface between the coexisting isotropic and nematic phases and this surface tension depends on the angle between the director and the plane of the interface; the variation of $\gamma$ is as high as $40 \%$ more when the nematic director is normal to the plane than when it is parallel to the interface. The number of atoms in the aggregate and the radius are related by

$$
\mathrm{N}=\frac{4 \pi \mathrm{r}^{3}}{3} \frac{\mathrm{N}_{\mathrm{A}} \rho}{\mathrm{M}}=\frac{4 \pi}{3} \mathrm{r}^{3} \rho
$$

Where $N_{A}$ is the Avogadro constant, $\rho$ the mass density, $M$ the molar mass, and $\bar{\rho}$ the number density. Combining both the above equations and differentiating with respect to either $N$ or yields values for the size of critical classical nucleus.

$$
\begin{aligned}
& \mathrm{N}_{\mathrm{c}}=\frac{32 \pi \gamma^{3}}{3 \rho^{-2} \Delta \mu^{3}} \\
& \text { and } \mathrm{N}=\frac{2 \gamma}{\rho \Delta \mu}
\end{aligned}
$$

An aggregate with the critical radius $r$ is the smallest structure for which the probability of growth is greater than the decay. Substitution of the critical radius in the first equation gives the energy barrier.

$$
\Delta_{\max } \mathrm{G}=\frac{4 \pi \mathrm{rr}_{\mathrm{c}}^{2} \gamma}{3}
$$

Assuming there is no additional activation barrier to formation of the critical immobilization surface, or MIP, that is that the maximum energy differences along the reaction path is equal to the Gibbs energy change required to form the critical nucleus, Then $E_{a c t}=\Delta_{\max } G$ and 
the rate of attach to the dispersed fractions of the imprinted polymer crosslinks depends exponentially on the adsorbed layer in contact.

In a model considers a multilayer surface with terraces of width $l$ on which atoms diffuse with a mean diffusion length $\lambda$. If the drugs which are deposited with flux $J$, re-evaporate from the step with a lifetime $\tau$, then the equilibrium concentration of the drug diffused can be evaluated. This model shows that for a given set of flux and diffusion parameters, a critical temperature exists below which template-binding imprint occurs. Above this temperature, the molecule accumulates on the MIP particle surfaces. This is based on step-flow growth. The difficulty of nucleating the new phase is expressed by the positive value of $\Delta_{\max } G$. It is unfavorable for selective sites to grow until the critical size is surpassed. The accretion and release of drugs from small particles leads to same distribution of small particles [81]. At thermal equilibrium the population of each size of microspherical MIP nanostructures leads to some distribution of small particles. Attachment and evaporation of atoms from the MIP nanostructures lead to constant conversion of different sizes. Random fluctuations in attachment occasionally push small cluster past the critical size, at which point they rapidly grow larger. The rate of formation of immobilized MIP surface onto the beads with the critical size determines the rate of formation of the new phase. On a surface, this rate is related to the rate at which template enantiomer molecules are bound to the original imprint site as well as the rate of surface diffusion to and formed steps and between each particle. In addition to energetic factors, nucleation can be affected by structural features. Steps and or elastic stain interactions can lead to preferential sites for template-monomer complex, which can have dramatic implications for structure formation.

Transport process as well as the critical nucleus size effect the evolution of layer morphology as material is added to the system, in particular, the evolution of the particle size distribution if the system is not following step flow growth. In nucleation regime, addition of material to the substrate leads to the formation of rough layers, which share the same mean size [82]. The number of nucleus changes but not their size distribution. In the growth regime, the island density no longer changes but the size of the interfaces increases. The mobility and coverage of drug molecule added to the imprinted cavity must be such that they are more likely to encounter and add to the cavities than to find another one and form a MIP-template interaction. The extent of their nucleation phase is, therefore, determined by the density of the MIP pore walls.

Because of the importance of transport process - the interplay between deposition rate, diffusion rate and the distance between the imprints - the critical nucleus size can be determined not only by thermodynamics, but rather by kinetic phenomena. At high coverage direct incorporation of transferring template and diffusion capture by stable imprints sites. At high coverage direct incorporation of the template enantiomer into the stable MIP-template complexes can also become significant [83]. The diffusion coefficient $D$ depends on the cluster as given by:

$$
\mathrm{D}=\mathrm{D}_{0} \exp \left(-\beta \mathrm{E}_{\text {diff }}\right)
$$

where $\beta=\left(k_{B} T_{s}\right)^{-1}$ is the relative coverage of stable clusters and $E_{d i f f}$ the diffusion activation energy. These equations demonstrate explicitly how the imprint coverage depends on kinetic parameters such as the transport diffusion rate of template, which is directly proportional to the growth rate and the temperature dependence of diffusion and detachment from the critical cluster. One consequence of the importance of transport dynamics is that the critical cluster size as well as the number of imprinted cavity can depend on the flux of incident template molecules. Furthermore, anisotropic diffusion, strain fields or anisotropic accommodation of the bound templates to the cavities can lead to the formation of anisotropic accommodation. In addition, any distribution of imprints that is created using kinetic control is subject to a kinetic frame work which the temperature raised sufficiently to facilitate diffusion in all directions.

\section{Design and engineered enantioselective MIP-based device}

Different approaches that can allow for a more efficient binding of the template molecule with an expanded recognition of the polymeric network have been proposed. This is of great significance for the development of an enantioselective MIP site inside the matrix or membrane or a dosage formulation consisted of a therapeutic agent that offers the properties in terms of binding affinity, capacity and diffusion. This means that only one of the two stereoisomers the eutomer (isomer of interest) - is required to be released for its therapeutic effect, while the other remains more firmly bound so it cannot exert any toxic effect. For the fabrication of imprinted sites as a mean to separate enantiomers and the interaction with the other chemicals in complex biological matrix and apply for recognition and selectively release of the specific enantiomer in the body, a number of a limitations still remain.

Transport across the biological membrane is essential. Compounds must be soluble enough in the lipid to get into the membrane, but not so soluble that they remain there. The extend of drug penetration in fact, is that, the imprinted site within the membrane pores is exploited to obtain very high selectivities, so site carrier membranes need to intimate membranes and strongly limit diffusion that is not dependent on carrier complexation. In previous studies the MIP system is one of the best performing facilitated transport membranes with respect to the enantiomeric separation of a pairs of mirror-imaged forms and selective transport upon dissolution phase of pharmaceutical formulations reported to date $[53,58,63]$. The selectivity behavior for imprinted enantiomer remarkably superior over non-imprinted enantiomer shows improvement with increasing cross-linking density and decreasing flux compared to the unmodified membrane. The enantioselectivity for template molecule increase significantly compared to analogous recognition separations in situ place on the biological barriers, which provides evidence for the existence of specific cavities in the device due to its contribution to the observed changes in chiral recognition properties and indicates that the extremely polar solvent media stimulates for chiral recognition. The mobile membrane proteins bearing electrical charges in a fluid/solid interface occur in viscous matrix a lateral force or diffusion [84], or, in other words this involves to forming dissipative structures powers by a concentration gradient of salt govern the difference of Donan membrane potential [85]. By molecular imprinting approach, the application of cationic drugs across epithelial layer of excised skin might have been due to a molecular effect by dissipative attractive forces inducing local gradients of the membrane potential and change in polarization. The effects of salvation on the electrostatic and van der Waals' interactions, are significance, giving rise to competitive effects of hydration and hydrophobic effect. There will be someloss of translational and rotational entropy on solution. The translational and rotational entropy loss on binding to receptor site thus is not very different between binding in gas phase and solution. Although individual van der Waals interactions are quite small, since they are common to all atoms, the net effect may become dominant in the overall template binding to the MIP inside a polymer system. However, upon binding and preferential configuration 
has a spreading of the perturbed frequency modes to both higher and lower frequencies. The latter will dominate the entropy contributions and may become significantly important. But internal rotations of the molecule and vibration due to dihedral angle variation are generally considered as more entropy-rich than all but the lowest-frequency vibration modes. However, molecular channel in proteins caused by specific ion conductivities in membranes [86], or as the electrophoresis that membrane proteins are electrically charged, moving in an applied field is considered [87]. The stability and selectivity translated of target binding result from the introduction of specific recognition cavities that contain intermolecular cavities capture and get the bioactive molecule or biologically important substance and release through the biological barrier that may be structurally translated into organization within organized cell membrane [86].

As above described, the lateral organization of membranes may be controlled externally e.g. by the adsorption of proteins. Thus, molecular self-organization is advantageous. The model holds for nature of individual distinct electrical potential and salt concentration having mobile charged conductivity that provides lateral and traverse transport combine together [87]. The deposition of small organic molecule in biological-receptor-substrate interactions is of great importance where adaptation is often required for regulation occurs. In this way depends on two fundamental assumptions: physicochemical properties of vehicle through the separating membranes obtained by these imprinted polymers together with membrane and biofunction of biological barriers, all of these that can indeed be altered to good sufficient as they can diffuse into from racemic compound of pharmaceutical active ingredients. However, we have to consider with the balance between rigidity and flexibility is of particular importance for the dynamic properties of the component, although this may deal some synthetic effort and the choices of suitable material, and often it requires clearly defined optimization of binding agents, thus yielding suitable interactions can be rearranged with complexity of substance binding sites directed towards the target species. However in the field of biomembranes in addition to the transport with integral proteins diffusing freely in a liquid/lipid bilayer it necessarily includes additional features appeared to interactions with cell skeleton too. In other words, this may be relevant to epithelial tissue, or otherwise abiotic membranes, and phase boundaries. Also, the fabricating nanostructures, more and more powerful methodologies provides the materials with highly complex functional architectures, strong potential to providing the adaptation and extend for 'smart' nanomaterials [88]. Supramolecular nanochemistry contributes deeply in the self-organization processes covering a wide range of self-processes determine the internal build up and the operation of entity as well as the interaction to the external environment through supramolecular nature. Such structural features enable important groups, essential for activity, to be suitably oriented in space so that drug interaction can take place. Any in situ-generated product is a good binder because it will have the same entropic and enthalpic factors that stabilized the complex ligand affinity, and they will add to the overall binding interactions among receptor and bound enantiomer template. The imprinted site as well as frame work porosity derived from format of mesoporous MIP microsphere of nanostructure within coating layer or core-shell having imprints on a shell layer, it plays an important role in achieving a highly shapeselective site generating from specific imprinted pockets. This approach represents the extent of the template-monomer complexation to three dimensions, and the control of functionality of functional monomer positioning by organizing within polymeric microenvironments [89]. With respect to improving recognition feature, capturing ability and chemical reactivity, yet leading the reactions occur within the template self-assembled binding site possessing reactivity and catalysis is crucial for the development of structural and reaction [90].

\section{Factor that influence the enantioselectivity}

A great deal of information concerning the stratum corneum, is the outermost layer of the skin, presents the principle barriers to passive diffusion of most molecules. The permeation of enantiomer template molecules through the stratum corneum is believed to proceed through the intercellular lipids. The extent of the optimal intermolecular physical forces for the enantioselective binding properties by the functional monomer is mainly governed by the chemical nature of each of the components in the imprinting mixture and the physical environment acting upon the polymerization process. These alternative approaches require the use of external energy to act as a driving force or a different $\mathrm{pH}$ gradient in order to enhance the template polymerization and microenvironment of the synthetic polymerization promote the selectivity [91]. Once we look at the subcutaneous that has a unique structure in which the dead corneocytes are cemented by intercellular lipids and glycoprotein structures (desmosomes) that are covalently bound to the epidermis. The possibilities stand out for potential improvement on these results so that adequate steric access to the imprinted sites by the peptide chains which are themselves attached to a truly vast, on a molecular scale, payload, namely glycoprotein. The conformation of the receptor assembly enables specific regions to be spatially oriented so that interactions may occur with complementary groups on the drug molecule, thereby initiating a biological response. In addition the lipid region consisted of lecithin which is the ammonium salt of choline connected to a phosphate group by an ester linkage. The nitrogen has a positive charge, as does the ammonium ion. As a consequence differences in the complexation between the enantiomers may be as small as $1 \%$ yet might be sufficient to cause a crossing of the selectivity, and implicating an inversion of the transport of the enantiomers. In all instances in which there is a drug and an MIP-ligand complex, the separation of enantiomers can never reach selectivity values that are as high as the intrinsic binding selectivity of the template-MIP; usually it is found with a neutral monomer and charged analytes and vice versa [92]. In cases, however, in which the MIP-ligand complex is made by the positive charge of a monomer and the unbound drug has a negative charge, an extremely high selectivity value can be attained under certain conditions. Strategies with favorable properties via molecular imprinting satisfying the following conditions required to operate as a carrier in the co-transport mechanism: (a) non-volatile and lipophilic - so that it will reside exclusively in a lipoidal membrane, (b) some surface activity - so that it adsorbs and desorbs at interfacial regions, and (c) exist in anionic form in order to ion-pair with cation at a specified $\mathrm{pH}$, i.e. $p K_{\mathrm{a}}$ of the acid should be less than $\mathrm{pH}$ of the system. To achieve a mass transfer of the template-MIP complexation formed between a charged free drug and the oppositely charged complex, in most cases multiple charged MIPs are required. At a particular amount of imprinted site at which both enantiomers diffuse with an equal but opposite effective transfer it is not at all a prerequisite for diffusional transport to occur as the result of charge neutralization and complexation. By carefully adjusting the amounts of imprint sites to the values close to the discontinuity even small intrinsic selectivities can be magnified to become large effective ones by a membrane [93].

In drug development, early information on human metabolism of a new drug is critical in predicting potential clinical drug, drug interactions and in selecting the appropriate animal species for toxicity studies. The major metabolic pathway may be shifted, depending on the 
drug concentration used. Good absorption is one of the most important criteria in selecting new drug candidates for development. Considering a partition equilibrium of enantiomers by an MIP as carrier is still valid. Thus, the carrier structure; complexation, aggregation or association and their distribution, is not significantly influenced by the partition process. If one can treat the MIP as a selector unit mimic, the binding affinity of the MIP-template aggregation and penetration of the bound template as the degree of partition into the system, or polymer. Its dependence on the partition coefficient of the enantiomer between the resultant membrane mass and MIP is then:

$$
\beta_{\mathrm{R}}=\frac{\mathrm{k}_{\mathrm{R}}}{1+\mathrm{k}_{\mathrm{R}}}(9)
$$

A dependence of the partition coefficient, $k$, (and the polymer) concentration, requires that the binding structure and their average aggregation and association particularly the partition coefficients, $k$ do not depend on the amount of the polymer. This optimum $k$ value again depends on the relation between the mass transfer kinetics of the free drug, by the imprint sites in a polymer $[53,94]$.

In biological systems, phospholipids form bilayer, in which the lipophilic tails line up against one another, forming a sheet like lamellar membrane with hydrophilic heads, by which phase changes can be induced at constant temperature by modulating chain packing. The MIP, as the recognition factor with predefined selectivity, exerts its influence by increasing the uptake of the targeted enantiomer of a preferred substance into a solid layer, and not by increasing the deposition of the substance. Differences in vehicle nature, tortuosity, and construction of a gel network will affect template binding with MIP in polymeric environment or the vehicle and thus its release rate. The basis for molecular memory lies in the formation of a chiral print molecule-functional monomer solution in the polymerization reaction. The more stable is the enantiomer print molecule-functional monomer complex, the greater will be the number, and fidelity of the resultant synthesized MIP products. Chirally imprinted MIPs, in principle, fulfill these major requirements and therefore may provide interesting prospects for the development of the enantioselective transport with target delivery by a means of molecular imprinting approach.

A single equilibrium has been considered, for complexation or partition; given that the diffusional template transport and separation of neutral and permanently charged enantiomers and MIPs can be described. The fixed $\mathrm{pH}$ values are considered or the $\mathrm{pH}$ ranges may be distant to the $\mathrm{pK}_{\mathrm{a}}$ values of weakly basic or weakly acidic groups in the enantiomers and or flexible mobile carriers. As any change in $\mathrm{pH}$ values that proximate to these $\mathrm{pK}$ values will have a big effect on the enantioselectivity. A similar treatment is required when combining complexation and partition equilibria as for instance when using an enantioselective MIP.

For weakly acidic and to weakly basic templates and MIPs, respectively it is important to note that throughout, the $\mathrm{pK}$ values are defined always in the direction of the deprotonation (dissociation of neutral weakly acidic groups and deprotonation of the protonated weakly basic groups); $\mu_{\mathrm{s}}^{\text {free }}$ and $\mu_{\mathrm{R}}^{\text {free }}=0$ ). The complexation constants of the neutral species are different from those of the corresponding charged species, i.e., $K_{S H}$ is unequal to $K_{S-}$ and $K_{S H+}$ is unequal to $K_{S}$ (and accordingly, for the $(S)$-enantiomer; the intrinsic selectivity changes when switching from a neutral to a charged species and can even be inverted. If the four species involved for the $(S)$-enantiomer are $\mathrm{SH}$ and R-MIP as well as SH-MIP and S-MIP, the complexation constants are $K_{S H}$ and $K_{R-}$ (all items are analogous for the $(R)$-enantiomer). The equilibrium constants for the dissociation of the free (noncomplexed) enantiomers, $K_{a}$ are equal, then the analogous equilibrium constants for the complexed enantomers $K_{a S}$ and $K_{a R}$ are different.

The $\mathrm{pH}$ is now an additional and important tool for optimizing separation. The equations allow one to calculate the effective mobility, selectivity and effective charge as a function of the two variables, polymer concentrations and $\mathrm{pH}$. The parameter values of the nondissociated species of both enantiomers at very low $\mathrm{pH}$ values at which the non-dissociated species are exclusively or far from being predominantly present, and vice versa, by determining the analogous constants for the dissociated species at high $\mathrm{pH}$ values at which the dissociated species are exclusively present.

\section{The accessibility of the binding site in vivo}

The receptor is defined a binding site with structural specificity but restrictive pharmacological deviation is to be preferred, although in any case the factors involved in drug binding at a stereospecific site are the same. Toward a flexible drug molecule, only a restricted conformation or set of conformations is likely to bind to the active receptor site. Alternatively, the rapid and straightforward layer synthesis also has the advantage of allowing for the efficient design of imprinted cavity with reversible binding and pronounced selectivity [95]. For percutaneous absorption by the use of MIPs, the intracellular pathway through the intercellular lipids, which water also penetrates via this pathway, in fact this represents one of important route of administration of pharmaceutical active ingredients and biopharmaceutical products [96]. Therefore, many approaches have been considered so as to increase entering at available accessed site after administration and percutaneous absorption of such compounds to explore and rich physical properties of soft two-dimensional materials, of which involves a large increase in controlled diffusion efficiency [97]. The largest impact of the biological sciences on nanotechnology may prove to be through the lessons one can learn from biology, rather than from directly employing the biological molecules themselves. To this end, it is important to support research on various aspects of biomimetics, including activities in which synthetic and biologically-based molecules are combined in a chimeric fashion. It can be seen that nature's biochemistry and pharmacy is usually very specific. However, when we tries to design a ligand to remove or mobilize a drug to produce a treatment, the best we can hope for is selectivity, i.e. there will inevitably be some sidereactions because more than the one desired enantiomer will be bound in the imprint cavity. Or, drug application to the skin is steadily used for local effects, also constructing dosage forms where passage through the skin into the systemic circulation.

The enantiomer difference in affinity was frequently found to be greater the higher the affinity of the more active enantiomer was, the so-called Pfeiffer's rule [98]. Most investigations have been directed towards the production of enantioselective imprints, achieved by means of this crucial stereochemically dependent interaction, to serve for the specific target selectivity of a transported enantiomer. The phenomenon of a selective release of therapeutic agents is derived entirely from specific interactions with the immobilized templatefunctional monomer sites. The eutomer of therapeutic agents are to be selectively transported across the skin a better therapeutic response might be expected relative to that obtained using a racemic mixture of the drug. Other ways of delivering the drugs to targeted tissues are therefore required, administered by topical administration in pharmacologically active stereoisomer predominantly. There are three ways of achieving by equilibrium competition for protein-enantiomer, 
decreasing the affinity of serum albumin, and stereoselective adsorption and trans-membrane delivery of desired enantiomers into target site for their therapeutic action.

The observed differences in chiral recognition between each of separate enantiomers through the specific imprinted cavity on the surface within the membranes with respect to differential interactions lie in differences in skin permeation rates. Measurement of transport rates, in a two phase system has been performed by which the test membrane of two immiscible phases is created at the surface of a sinter and solute transfer across the unstirred liquid and interfacial region of the stirred measured: thinner filter $(150 \mu \mathrm{m})$ replaced, say the 3 mm-membrane, result interfacial transfer became more significant and steady state fluxes were obtained rapidly [99]. There have been reported on the interface layers led to differences in stereomeric recognition of chiral molecules by an imprinted polymer surface [100]. The advantages of a greatly enhanced rebinding and accessibility due to having the intrinsic underlying feature of the surface, overcome the inherent thermodynamic hurdles associated with MIPs and allow for improved mass transport characteristics. The introduction of imprinted cavities acted an adsorbed molecule distributed onto surfaces can facilitate transport across a membrane, for more control of the efflux of the required enantiomer. The slightly removed drug templates from a smoother surface from many tiny particles surrounded with imprinted-polymer than polymeric environment, resulting in reduced force of adhesion between functional group of both binding entities providing reduction of disturbance of the binding sites and optimizing an appropriate process of controlled drug release [101].

The permeation of topically applied chemical across the skin has been subjected of considerable interest, both in the screening of candidates for topical and transdermal delivery and for the evaluation of cutaneous toxicity. Therefore, the tailored release of a drug continues to be a challenging aspect for artificial recognition systems in vivo. In addition to offering measurement of skin diffusion across excised human skin appears generally to correlate well with in vivo data [102]. A number of animal models have been used to measure percutaneous adsorption in vitro [103]. Bioavailability may be assessed by the determination of the induced clinical response which, because it often involves an element of subjective assessment, makes quantification difficult. Measurement of drug concentrations at the receptor site is not feasible; therefore, the usual approach is the determination of plasma or blood levels as a function of time, making the implicit assumption that these concentrations correlate directly with the clinical responses. An invitro-invivo correlation (IVIVC) should be evaluated to demonstrate that predictability of the in vivo performance of a drug product from its in vitro dissolution characteristics is maintained over a range of in vitro dissolution release rates and manufacturing changes. Developing of IVIVC is to establish a predictive mathematical model describing the relationship between an in vitro property and a relevant in vivo response, the proposed evaluation approaches focus on the estimation of predictive performance or, predictive error. Solubility and diffusion in the vehicle are factors influencing the delivery rate of enantiomers from racemic compounds. Further, any stereospecific interaction may be subtle and readily overwhelmed if diffusant concentration is greater than the capacity of the skin to differentiate between stereoisomers. As in vitro experiments are useful tool during the development of dermal preparations. Although it seem feasible the IVIVC approach has been utilized to correlate skin permeation data to the in vivo drug profiles, however standardization of experimental conditions are highly needed, for instance the method, the calculated cumulative fraction of drug absorbed at different time [104]
On the basis of the benefits of the molecular recognition based on molecularly imprinted polymerization as abovementioned, were developed for the discrimination within device for chiral recognition applications and biomedicine. Owing to the high efficiency of the method, offering possibility of creating tailor-made recognition sites, new ways were opened towards a better understanding of the various enantiomer selective release effects operative in aqueous and under conditions mimicking physiological environment. The self-organization of channels indicates an evidence of information in physical features of the membrane, i.e. protein, diffusion, electrophoresis, membrane potential, channel conductance, may be related to enourmous biological features [105]. Such interactions must be considered in terms of partitioning and diffusion limitation, the provision of interactive targets for the antimicrobial that compete locally with those associated with the target organisms (reactiondiffusion limitation), and the retention and organization of druginactivating enzymes (enzyme-mediated reaction-diffusion limitation). The considerations are nowadays inherent in the pharmacokinetic modeling of the bioavailability of drugs in animals and humans in the experiments proceeding with individual part or in whole body [106].

Skin metabolism has important implications in the pharmacological and toxicological aspects of therapeutic agents that come in contact with skin. Various aspects of the metabolism of pharmaceuticals and xenobiotics in the skin have been studied, and it has been shown that skin contains various enzymes including butyrylcholinesterase, arylesterase and acetylcholinesterase which are capable of hydrolyzing different esters. Despite genetic polymorphism in drug metabolism is undesirable and can at times be problematic. The different enzyme polymorphisms in drug metabolism and an inherited deficiency of different drug-metabolizing enzymes could occur simultaneously on the basis of probability. Genetic variants are known not only for butyrylcholinesterase, but also for arylesterase [107]. However, it should be emphasized that even if a large proportion of the metabolism of a compound is subject to genetic polymorphism, this should not influence its development as a drug. A catalytic (i.e. enzymatic) reaction could lead to severe therapeutic penetration reduction, provided that the turnover of substrate by the enzyme was sufficiently rapid. It has been noted that cutaneous stereoselective hydrolyses that was superior over by plasma and skin homogenate occurred. The stereoselectivity on nature and biological function of the skin epidermis since the reaction-diffusion limitation properties of the polymer matrix of biomaterial could be significantly enhanced if it contained extracellular enzymes that were capable of degrading the diffusing substrate [108]. In membrane-bound receptors, a wide variety of ligands, the peptide and glycoprotein, the biogenic amines are associated coupling to the enzyme. Moreover, transport processes are involved with either electrical potential or nonreactive channels, which physical features of membrane is hypothesized to be associated to biological features of slim tiny living organism and nanoparticles can pass through. The generating of imprinted material having high specific recognition with mimicking biological enzymes, and that should have similar chemical groups to those occurring in natural enzymes, even though the efforts have to be paid resulted in enzyme activity, but there is need to make the MIPs operate more effectively [109]. On the other hand, transport in membranes by charged channel condensation is believed to involve directly in electrophoresis in self-consist coupled the membrane potentials to space and local conductivity derived from dynamics of channel density which controlling of morphogenesis appearance by well correlation in molecular and global properties of biological significance regardless to channel density, reversal potentials and cell size [110] 


\section{Mechanism to enhance percutaneous drug delivery by MIPs}

The partitioning of a drug, defined as the ratio of concentrations between the vehicle and the membrane at equilibrium, depends on both the drug and the membrane's characteristics and which probe and understand with more molecular detail is feasible when the examining by spectroscopic method [111] or measurement by interfacing the MIP layer with a biological barrier, or even a transduce for senor measurement [66]. Whereas the mechanism of the phase behavior was governed by repulsive electrostatics and attractive entropic forces: a desirable amount of membrane bases was needed to reach the critical concentration required for phase separation, shifting the phase equilibrium into the region of isotropic-chiral nematic phase coexistence between chiral MIP material and membrane component. In Figure 4 , it is a sketch of the ordered arrangement of the therapeutic agents for specific adsorption on the imprinted layer as well as non-specific adsorption showing the specific structure of adsorbed drug depends on the balance between adsorption and adsorption energy of the imprint [112]. The ordering of water molecules at an insulator surface responds to the $\mathrm{pH}$ medium. The changing of $\mathrm{pH}$ the net charge on the polymer surface determines order water layer and its orientation. Because lateral compression of an ordered array of specific functional groups at the surface yields pressure/area isotherms relating to intermolecular interactions molecular species interacting with the respective MIP present at the solid-liquid interface. This provides structural surface in vacuum and the surface tension of solid/liquid interface on the permeation of a drug loaded on the functional material and non-specific layer across skin. This represents the areas of intriguing and rather futuristic field of chemistry, which perspective lie, of course in the long range. By the use of molecular non-covalent imprinting technique led to the consequence of development in molecular and supramolecular science and chemical biology of chirally active compounds in live cells which offer feasibility of perspectives at the frontriers of chemistry with physics and biology [113]. The following evidence obtained may also be evaluated in the view of developing structure and biomimetic properties to obtain substrate-specific MIPs make the possible future uses for MIPs that would perform highly selective functions of recognition, transformation, transfer, regulation and communication, and allow signal and information process, it is really a challenging task

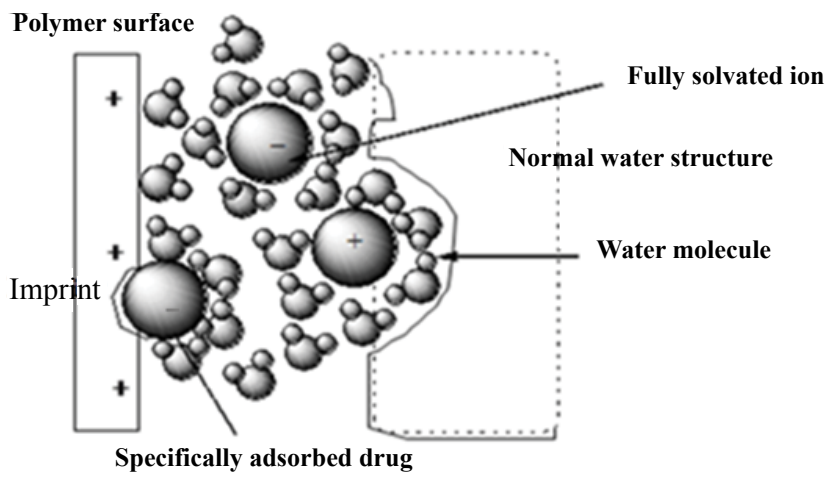

Figure 4: A model of the solid/liquid interface with specific adsorption on the imprinted layer and non-specific adsorption of the substrate, showing the specific structure of adsorbed drug depends on the balance between adsorption and adsorption energy of the imprint The structure of intact water layer near polymer surface is formed (dash line) which is influenced by the polar anchoring of the monolayer to the interface between polymer surface and adsorbed species in case of specific adsorption on the polymer surface and non-specific adsorption, respectively [112]
$[114,115]$. This implies operation via intermolecular interactions and incorporation of the complex dimension into recognition events which analogies with features of smart systems, thus linking processes of artificial intelligence and molecular behavior.

As the conceptual framework has been progressively laid down and very active task on molecular recognition, catalysis and transport, together with extension to molecular surfaces and molecular assemblies, is building up a vast body of knowledge on molecular behavior at the supramolecular level. Studies have shown that each of three key lipid classes is required for normal barrier function. For example, selective inhibition of either cholesterol or fatty acid, or ceramide synthesis in the epidermis delays barrier recovery rates after barrier perturbation of hairless mouse skin in vivo. This demonstrates that the possibility of species differences in transdermal enantioselectivity [116]. It holds promise to create artificial materials that have chemical properties or act as the potential of a selective inhibitor derived from MIP when applied with disruption agent of the barrier [117]. Regardless of the specific mechanism involved in the activation or deactivation of an enzyme, the overall result is the same which this leads to change in the treatment for agents used in the treatment of some of skin diseases bacterial, fungal, parasitic, and viral infections and chronic diseases such as cancer [118]. A change in receptor shape (i.e. protein, peptides) leads eventually to the activation (or deactivation) of enzymes. Since enzymes can catalyze the reaction of a large number of molecules, there is an amplification of the signal. Substantial biotransformations have been observed during the absorption process of a pair of stereoisomer with difference in stereospecific interaction. Sometimes drug designed against a specific target become less effective over time. Since cells have a highly complex system of signaling mechanisms, it is possible that the blocked of one part of that system could be by-passed. With cutaneous drug metabolizing enzymes, have an imperative role in the barrier function of the skin, any chemicals that are applied to be the surface of the skin will, during the course of penetration and translocation through this organ, be exposed to various biotransformation systems [119]. However particles pass through the film, they leave sensitized tracks where the chemical bonds break; the role of binding in hydroxyl groups on the structure is known which they are commonly involved in hydrogen bonding. The thought is that the proton of the hydroxyl group is involved in the hydrogen bond to the receptor and if it is removed the hydrogen bond is lost. Converting such a group to methyl ether or an ester is straight forward and will usually destroy or weaken such a bond. With molecules or small particles larger than the rated pore size the chemical bonds of these molecules break, will be they usually operate of osmosis if exceed the osmotic several salts as a function $\mathrm{NaCl}$ will have an effect [120].

As a flourish interest of applications of MIPs in dermal drug delivery, mechanism of biochemical pathways and identifying drugenzymes in the skin responsible to stereoselective events of chiral pharmaceutical substances is of importance that play important role in drug discovery research [121]. Metabolism by the skin can both reduce the local and systemic exposure to potentially toxic species and also lead to the generation of reactive species which may bind to skin proteins. Molecular imprinting which represents a novel method that are valuable tools for pharmacokinetic studies and even searching of drug candidates; as selective and potent inhibitors for skin and other metabolic enzymes this is valuable facilitating drug design and speed up in development and discoveries of drug [31,122]. They enable the identification of cutaneous enzymes that are responsible for drug metabolism when they come to contact the skin and can be applied to testing in tissue preparations [31]. The latter composite membranes 
could be developed toward tailored separation membranes, e.g. using MIP microgel as fixed or even mobile carriers, or toward catalytically active membrane-based on the immobilization of enzyme-mimicking MIPs. Membrane-immobilized proteins, receptor sites density may even be higher for MIP layers. These represent a crucial challenging in biological evolution and present a promising to chemistry. Furthermore, selective inhibitors can be used to elucidate drug-drug interactions and for the identification of enzyme polymorphism. Biochemical approaches via molecular imprinting technologies may cause a further boost in drug delivery, primarily attributed to protection form stereoselectively or selective metabolizing enzyme and to alter permeability barrier as feasible approach. The scientific design of imprinted materials for the delivery of the drugs are available and even drug candidates in biological screening test, enhancing transdermal/ transcutaneous delivery of pharmaceutical agents, provides that potentially develop safer, effective alternatives to drugs and minimize potential side effects.

\section{Conclusion and Remarks}

The development of new, highly selective chiral MIP for active and selective bioactive agents or biopharmaceutical substance recognition selectivity, has evolved to a skill integrating many disciplines such as organic chemistry, physical chemistry and biochemistry, closely interlinked in biology in its widest understand and wisdom. As such, modern advances in material sciences are now predicated upon the rationale strategies that create tailored pre-defined recognition sites for a specific target and engineered to have characteristics that mimics to normal biological functions. To obtain the desired information about the analyte molecule with a certain degree of confidence, it is necessary to develop efficient methods. In concert with combinatorial approach, the strategies of integration of molecule-imprinted polymer functionalization within a biopolymer is also providing opportunities of improving bioavailability of desired enantiomer of chiral pharmaceutical active compounds to overcome the limitation of biological barriers. In order to achieve the performance goals, further improvements of the preparation of surface immobilized MIP on nanostructures, or thin layer will be most effective approach. However any additional biological responses from having interfacial contact with the membrane system must be considered, particular for metabolized enzymes and suppression of the pathological defense mechanisms e.g. any immunoresponse and/or complement activation, yet at the same time produce a minimum cell toxicity. Hence emphasis on chemistry is consistent with the notion that recognition and specificity are usually characteristic motifs used throughout biology. Importance of analysis method is required for a molecular level and network characterizations of such materials. Increasingly the chemical

\section{Guidelines}

The scientific design for the implement of bioanalogous recognition abilities into a material to elucidate the forces that control guest interactions.

Design and creation of a recognition cavity is especially important, to implement the functionality of necessary stereochemical feature in artificial materials, and the discriminatory capacity of the specific sites.

The chemical engineering design of recognition system influences on the physicochemical properties of re-uptake and release of the initial stereoisomer template in a polymeric environment are needed to overcome for limitations.

The generating MIP material to achieve appropriate orientation at a catalytic of appropriately aligning with either the active or stabilization domain, yet rotational position and assist to pinhole compounds of different molecular entities but sharing a common target.

Parameters for chiral recognition by MIP-based matrix relate to: resolution, control of release and the robustness of the designed systems.

The ability to discriminate chirally by an imprinted chiral agent is complementary principles concern the charge distribution at the interface of the interacting molecules and the spatial structural arrangement.

Tighter binding (higher affinity, $K$ ) between one enantiomer and the functional monomer might be a source of reduced mass transfer and thus a slightly higher mobility of the complex.

The selectivity maximum to a higher concentration of the selective site when the template-MIP complex was decreasing, conferring to the absence of a differentiable maximum value at finite concentrations when the effects of association of the template species or the bound MIP were zero.

Molecular imprinted receptor strategy
The biomaterials and self-assembly of the complementary functional groups by polymerizing monomeric components containing a given template into a confined entity as a result of direct the generation of organized functional structures in equilibrium conditions.

The creating molecular recognition in material, where combined approach to the use of a material can selectively recognize and respond to variations in the analyte concentrations and trigger a controlled level of active determined by this molecular recognition event.

The mechanisms, of the extent and consequences of receptor co-localization and inter-receptor communication may significantly enhanced the understanding how a protein receptor and enzyme activity in their skin and elicit luminescence or fluorescence The presence of the fluorescent MIPs having a fluorophore stemming in the imprinted cavities make them amenable to mechanistic scrutiny by fluorescence spectroscopic techniques could be visualize through skin.

Growing the nano level to the meso level and the macro level with internal structural organization for MIP nanostructure incorporating within support membrane, template binding to imprinted sites can either change the pore network, therefore altering membrane permeability.

A certain thickness of membrane for optimizing the process and mismatching of the membrane perturbation energy that will not affect the functionalization of the selective materials. And, the chiral molecularly imprinted microspheres are shown to have distinctly different responses, and alterations of the cell surface in addition to those obtained by adding the parent compounds.

For stereoselective and trans-membrane transport The rationale for designing the binding agents involves the therapeutic effect of its administration arises from an increase in the plasma concentration in body compartments and this increase is fostered by the desired enantiomer found in plasma that can diffuse into fluid.

Nuclear magnetic resonance (NMR)

Characterization of recognition ability of MIPs
Fourier transform Infrared spectroscopy (FT-IR)

Fluorescence spectroscopy

Table 1: The guidelines for the construction of a synthesized recognitive network for potential application for dermal drug delivery. 
scientist is seeking ways to physically control biological responses related to a greater understanding of the various understanding of the different biological process in which receptors at membrane surface are involved, affecting the delivery of drugs. Methods have been developed to fabricate biopolymers that biopolymer-based products have been shown to link to vital interactions between polymers and biological systems, and that cover a wide variety of differences in bioactivity, yet produce a method for new processing. Material and polymer-tissue interactions indicate that the precise tailoring of physicochemical properties will be essential for future progress in the chiral recognition of drugs. Molecular imprinting has interesting implications for chiral drug delivery which is used as a model to study a means for administering single enantiomers from a racemate delivery through the skin to the circulation. It is a possible way to direct a flexible functionality in a minor abundant conformation adaptability at which synthesizing of recognition site through native interactions of chiral print molecules that are complementary to the imprinting mixture. The development of this approach allowing rapid characterization of chemical and pharmaceutical properties of the biologically important compounds represents an exciting new advance in the pharmaceutical sciences, which not only can lead to an improved understanding of the biological barriers that limit the effectiveness of drug candidates, but which can also expedite the process of optimization of imprinted polymer materials to produce dermal or transdermal drug delivery system (Table 1).

\section{Acknowledgements}

Financial support is gratefully acknowledged from the Higher Education Research Promotion and National Research University Project of Thailand, Office of the Higher Education Commission and the Nanotechnology Center (NANOTEC), NSTDA, Ministry of Science and Technology, Thailand, through its program of Center of Excellence Network and ASEA-Uninet, University of Vienna, Austria and Prof. Franz L. Dickert for providing preprint versions from his scientific works. The author would like to thank Prof. Gary P. Martin for his helpful comments and Dr. Brian Hodgson for his assistance with the English.

\section{References}

1. Ye L, Yu Y, Mosbach K (2001) Towards the development of molecularly imprinted artificial receptors for the screening of estrogenic chemicals. Analyst 126: $760-765$.

2. Pasteur $L$ (1901) On the asymmetry of naturally occurring organic compounds the foundations of stereochemistry. In: Memoirs by Pasteur, ban't Hoof, le Bel and Wislicenus (Ed. Richardson GM), American Book Company, New York: $1-33$.

3. MacBeath G, Schreiber SL (2000) Printing proteins as microarrays for highthroughput function determination. Science 289: 1760-1763.

4. Yoshimatsu K, Reimhult K, Krozer A, Mosbach K, Sode K, et al. (2007) Uniform molecularly imprinted microspheres and nanoparticles prepared by precipitation polymerization: the control of particle size suitable for different analytical applications. Anal Chim Acta 584: 112-121.

5. Datta S, Grant DJ (2004) Crystal structures of drugs: advances in determination prediction and engineering. Nat Rev Drug Discov 3: 42-57.

6. Seibel J, Allemann O, Siegel JS, Ernst KH (2013) Chiral conflict among different helicenes suppresses formation of one enantiomorph in $2 \mathrm{D}$ crystallization. $\mathrm{J}$ Am Chem Soc 135: 7434-7437.

7. Piletsky S, Turner A (2006) Molecular Imprinting of Polymers. Landes Bioscience: Georgetown, Texas: 1-220.

8. Yilmaz E, Haupt K, Mosbach K (2000) The Use of Immobilized Templates-A New Approach in Molecular Imprinting. Angew Chem Int Ed Engl 39: 21152118.

9. Fischer E (1894) Einfluss der Configuration auf die Wirkung der. Enzyme. Ber Dtsch Chem Ges 27: 2985

10. Goldberg M, Langer R, Jia X (2007) Nanostructured materials for applications in drug delivery and tissue engineering. J Biomater Sci Polym Ed 18: 241-268.
11. Haupt K, Mosbach K (2000) Molecularly imprinted polymers and their use in biomimetic sensors. Chem Rev 100: 2495-2504.

12. Hayden O, Mann KJ, Krassnig S, Dickert FL (2006) Biomimetic ABO bloodgroup typing. Angew Chem Int Ed Engl 45: 2626-2629.

13. Maier NM, Lindner W (2007) Chiral recognition applications of molecularly imprinted polymers: a critical review. Anal Bioanal Chem 389: 377-397.

14. Byrne ME, Hilt JZ, Peppas NA (2008) Recognitive biomimetic networks with moiety imprinting for intelligent drug delivery. J Biomed Mater Res A 84: 137147.

15. Andersson LI, MÃ $1 / 4$ ller R, Vlatakis G, Mosbach K (1995) Mimics of the binding sites of opioid receptors obtained by molecular imprinting of enkephalin and morphine. Proc Natl Acad Sci U S A 92: 4788-4792.

16. Ahuja S (1997) In: The impact of stereochemistry on drug development and use, Edited by Aboul-Enein H, Wainer IW. Wiley-Interscience Publication, John Wiley \& Son, Inc, New York.

17. Ariens EJ (1986) Chirality in bioactive agents and its pitfalls. Trends in Pharmacological Sciences 7: 200-205.

18. Ariens EJ (1991) Racemic therapeutics--ethical and regulatory aspects. Eur $J$ Clin Pharmacol 41: 89-93.

19. Drayer DE (1988) The early history of stereochemistry. In: Drug stereochemistry, analytical methods and pharmacology, Edited by Wainer IW, Drayer DE. New York and Basel: Marcel Dekker, Inc: 3-29.

20. Eriksson T, Bjorkman S, Roth B, Fyge A, Hoglund P (1995) Stereospecific determination, chiral inversion in vitro and pharmacokinetics in humans of the enantiomers of thalidomide. Chirality $7: 44-52$

21. Ariens EJ (1984) Stereochemistry, a basis for sophisticated nonsense in pharmacokinetics and clinical pharmacology. Eur J Clin Pharmacol 26: 663668.

22. Ariens EJ (1986) Stereochemistry: a source of problems in medicinal chemistry. Med Res Rev 6: 451-466.

23. Ariens EJ (1987) Implications of the neglect of stereochemistry in pharmacokinetics and clinical pharmacology. Drug Intell Clin Pharm 21: 827829.

24. Suedee R, Srichana T, Martin GP (2000) Evaluation of matrices containing molecularly imprinted polymers in the enantioselective-controlled delivery of beta-blockers. J Control Release 66: 135-147.

25. Vlatakis G, Andersson LI, Muller R, Mosbach K (1993) Drug assay using antibody mimics made by molecular imprinting. Nature 361: 645-647.

26. Wulff G (1995) Molecular imprinting in cross-linked materials with the aid of molecular templates-A way towards artificial antibodies. Angewandte Chemie International Edition in English 34: 1812-1832.

27. Andersson LI, Muller R, Vlatakis G, Mosbach K (1995) Mimics of the binding sites of opioid receptors obtained by molecular imprinting of enkephalin and morphine. Proc Natl Acad Sci U S A 92: 4788-4792.

28. Kempe M, Fischer L, Mosbach K (1993) Chiral separation using molecularly imprinted heteroaromatic polymers. J Mol Recognit 6: 25-29.

29. Ye L, Mosbach K (2008) Molecular imprinting: synthetic materials as substitutes for biological antibodies and receptors. Chem Mater 20: 859-868.

30. Mosbach K (2001) Toward the next generation of molecular imprinting with emphasis on the formation, by direct molding, of compounds with biological activity (biomimetics) Analytica Chimica Acta 435: 3-8.

31. Yu Y, Ye L, Haupt K, Mosbach K (2002) Formation of a class of enzyme inhibitors (drugs), including a chiral compound, by using imprinted polymers or biomolecules as molecular-scale reaction vessels. Angew Chem Int Ed Engl 41: 4459-4463.

32. Suedee R (2013) Novel strategic innovations for designing drug delivery system using molecularly imprinted micro/nanobeads. Int J Pharm Sci Rev Res 20: $235-268$.

33. Shea KJ, Yan M, Roberts MJ (2002) Molecularly imprinted materials-sensors and other devices Materials Research Society Symposium Proceedings 723 : 5-15.

34. Fasco MJ, Principe LM (1982) R- and S-Warfarin inhibition of vitamin K and vitamin K 2,3-epoxide reductase activities in the rat. J Biol Chem 257: 4894 4901. 
35. Andersson LI (2000) Molecular imprinting for drug bioanalysis. A review on the application of imprinted polymers to solid-phase extraction and binding assay. J Chromatogr B Biomed Sci Appl 739: 163-173.

36. Lieberzeit PA, Rehman A, Iqbal N, Najafi B, Dickert FL (2009) QCM sensor array for monitoring terpene emissions from odoriferous plants. Monatsh Chem 140: 947-952.

37. Pregenzer JF, Alberts GL, Bock JH, Slightom JL, Im WB (1997) Characterization of ligand binding properties of the 5-HT1D receptors cloned from chimpanzee, gorilla and rhesus monkey in comparison with those from the human and guinea pig receptors. Neurosci Lett 235: 117-120.

38. Sontimuang C, Suedee R, Dickert F (2011) Interdigitated capacitive biosensor based on molecularly imprinted polymer for rapid detection of Hev b1 latex allergen. Anal Biochem 410: 224-233.

39. Hillery AM, Lloyd AW, Swarbrick J (Eds) In: Drug delivery and targeting for pharmacists and pharmaceutical scientists, Taylor \& Francis, London and New York: $1-395$

40. Elisseeff JH, Fischbach C, Freier T, Garcóa AJ, Mooney DJ, et al. (2006) Polymers for regenerative medicine, In Advances in polymer sciences, Werner C (ed) Springer - Verlag Berlin Heidelberg New York: 1-227

41. Robert E, Armstrong RE, Drapeau MD, Loeb CA, Valdes JJ (2010) Bioinspired innovation and natural security. National Defense University Press, Washington DC: $1-374$

42. Moghimi SM, Hunter AC, Murray JC (2005) Nanomedicine: current status and future prospects. FASEB J 19: 311-330.

43. Klefenz H (2002) Industrial Pharmaceutical Biotechnology. Wiley-VCH Verlag $\mathrm{GmbH}: 1-297$.

44. Griffin JP (2009) The textbook of pharmaceutical medicine. (6thedn), John Wiley \& Sons, London: 81-136.

45. Vasapollo G, Sole RD, Mergola L, Lazzoi MR, Scardino A, et al. (2011) Molecularly imprinted polymers: present and future prospective. Int $\mathrm{J}$ Mol Sci 12: $5908-5945$

46. Dan R, Wang Y, Du L, Du S, Huang M, et al. (2013) The synthesis of molecular imprinted chitosan-gels copolymerized with multiform functional monomers a three different temperatures and the recognition for the template ovalbumin Analyst 138: 3433-3443.

47. Lee KJ, Kim DS, Kim BS (2007) Biodegradable molecularly imprinted polymers based on poly(e-caprolactone). Biotech Bioprocess Eng $12: 152-156$.

48. Borck A, Bunge A, Biela S (2012) Implantable theranostic article. US20120123235.

49. Ali M, Byrne ME (2013) Controlled and extended delivery of hyaluronic acid and comfort molecules via a contact lens platform. US8388995.

50. Mayes AG, Whitcombe MJ (2005) Synthetic strategies for the generation of molecularly imprinted organic polymers. Adv Drug Deliv Rev 57: 1742-1778.

51. Whitcombe MJ, Rodriguez ME, Villar P, Vulfson EN (1995) A new method for the introduction of recognition site functionality into polymers prepared by molecular imprinting: Synthesis and characterization of polymeric receptors for cholesterol. J Am Chem Soc 117: 7105-7111.

52. Sellergren B, Ekberg B, Mosbach K (1985) Molecular imprinting of amino acid derivatives in macroporous polymers : Demonstration of substrate- and enantio-selectivity by chromatographic resolution of racemic mixtures of amino acid derivatives. Journal of Chromatography A 347: 1-10.

53. Dickert FL, Hayden O, Bindeus R, Mann KJ, Blaas D, et al. (2004) Bioimprinted QCM sensors for virus detection-screening of plant sap. Anal Bioanal Chem 378: $1929-1934$

54. Sellergren B (2001) The non-covalent approach to molecular imprinting. In Molecularly imprinted polymers: Man made mimics of antibodies and their application in analytical chemistry. Sellergren B (ed) Amsterdam: Elsevier Science B: 113-184

55. Poma A, Turner AP, Piletsky SA (2010) Advances in the manufacture of MIP nanoparticles. Trends Biotechnol 28: 629-637.

56. Silvestri D, Cristallini C, Ciardelli G, Giusti P, Barbani N (2005) Molecularly imprinted bioartificial membranes for the selective recognition of biological molecules. Part 2: release of components and thermal analysis. J Biomater Sc Polym Ed 16: 397-410.
57. Koshland DE (1958) Application of a Theory of Enzyme Specificity to Protein Synthesis. Proc Natl Acad Sci U S A 44: 98-104

58. Waris T, Rechardt L (1977) Histochemically demonstrable catecholamines and cholinesterases in nerve fibres of rat dorsal skin. Histochemistry 53: 203-216.

59. Persin Z, Stana-Kleinschek K, Foster TJ, van Dam JEG, Boeriu CG, et al. (2011) Challenges and opportunities in polysaccharides research and technology: The EPNOE views for the next decade in the areas of materials, food and health care. Carbohydrate Polymers 84: 22-32.

60. Andersson LI, Nicholls IA, Mosbach K (1994) A versatile tool for the preparation of separation materials of predetermined selectivity. In: Highly selective separations in biotechnology, Edited by Street G (Chapter 9) Glasgow: Blackie Academic \& Professional: 207-225.

61. Deilami SA, Abdouss M, Seyedib SR (2010) Synthesis and characterization of molecularly imprinted polymer for controlled release of tramadol. Central European Journal of Chemistry 89: 687-695

62. Yin J, Cui Y, Yang G, Wang $H(2010)$ Molecularly imprinted nanotubes fo enantioselective drug delivery and controlled release. Chem Commun (Camb) 46: 7688-7690.

63. Ahmed S, Imai T, Yoshigae Y, Otagiri M (1997) Stereospecific activity and nature of metabolizing esterases for propranolol prodrug in hairless mouse skin, liver and plasma. Life Sci 61: 1879-1887.

64. Shea KJ, Sasaki DY (1991) An analysis of small-molecule binding to functionalized synthetic polymers by 13C CP/MAS NMR and FT-IR spectroscopy. J Am Chem Soc 113: 4109-4120.

65. Sellergren B, Shea KJ (1993) Chiral ion-exchange chromatography. Correlation between solute retention and a theoretical ion-exchange model using imprinted polymers. J Chromatogr A 654: 17-28.

66. Dickert FL, Lieberzeit P, Hayden O (2004) Molecularly imprinted polymers for mass sensitive sensors-from cells to viruses and enzymes. In: Molecular imprinting of polymers. Piletsky S, Turner A, Nicholls I (eds), Eurekah.com Chapter 8: 1-15.

67. Bagatolli L, Kumar PBS (2009) Phase behavior of multicomponent membranes: Experimental and computational techniques. Soft Matter 5: 3234-3248.

68. Miyazaki K, Kaiho F, Inagaki A, Dohi M, Hazemoto N, et al. (1992) Enantiomeric difference in percutaneous penetration of propranolol through rat excised skin. Chem Pharm Bull (Tokyo) 40: 1075-1076.

69. Daniels DR, Turner MS (2007) Diffusion on membrane tubes: a highly discriminatory test of the Saffman-Delbruck theory. Langmuir 23: 6667-6670.

70. Jacob R, Krishnan BS, Venkatesan T (2004) Pharmacokinetics and pharmacodynamics of anesthetic drugs in pediatrics. Indian J Anaesth 48: 340 346.

71. Higuchi WI, Fox JL, Knutson K, Anderson BD, Flynn GL (1985) In: Directed drug delivery. Borchardt RT, Repta AJ, Stella VJ, Exp Bio Med 7: 97-117.

72. Minuth WW, Denk L, Roessger A (2009) Gradient perfusion culture-simulating a tissue-specific environment for epithelia in biomedicine. Journal of Epithelial Biology and Pharmacology 2: 1-13.

73. Ekinci D (2012) Protein flexibility and coiled-coil propensity: New insights into Type III and other bacterial secretion system. In Biochemistry. Intechopen, Rijeka, Croatia: 1-39.

74. Ren K, Zare RN (2012) Chemical recognition in cell-imprinted polymers. ACS Nano 6: 4314-4318.

75. Quignot N (2013) Modeling bioavailability to organs protected by biological barriers. In Silico Pharmacology 1: 1-8.

76. Azadbakht B, Hadad K, Zahmatkesh MH (2009) Responsive verification of dose rate and time dependence of PAGAT polymer gel dosimeters by photon beams using magnetic resonance imaging. J Phys 164: 1-10.

77. Santin M, Phillps GJ (2012) Biomimetic, bioresponsive and bioactive, an introduction to integrating materials with tissues. John \&Willey, New York: $1-249$.

78. Tomellini R, Faure U, Oanzer O (2005) Technology Platform Strategic, Research Agenda for Nanomedicine, Tyndall National Institute, Belgium: 1-45.

79. Rotello V Thayamanavan S (2010) In: Molecular recognition and polymers, Control of polymer structure and self-assembly. John Wiley \& Son, New York: $1-493$. 
80. Perez M, Dumont M, Acevedo-Reyes D (2008) Implement of classical nucleation and growth theories for precipitation. Acta Materialia 56: 2119-2132.

81. Wei S, Jakusch M, Mizaikoff B (2007) Investigating the mechanisms of 17betaestradiol imprinting by computational prediction and spectroscopic analysis. Anal Bioanal Chem 389: 423-431.

82. Arrua RD, Strumia MC, Igarzabal CIA (2009) Macroporous monolithic polymers: preparation and applications. Materials 2: 2429-2466.

83. Václavík J, Kačer P, Kuzma M, Cervený L (2011) Opportunities offered by chiral $\eta^{6}$-arene/ $\mathrm{N}$-arylsulfonyl-diamine-Rull catalysts in the asymmetric transfer hydrogenation of ketones and imines. Molecules 16: 5460-5495.

84. Singer SJ, Nicolson GL (1972) The fluid mosaic model of the structure of cell membranes. Science 175: 720-731.

85. HODGKIN AL, HUXLEY AF (1952) A quantitative description of membrane current and its application to conduction and excitation in nerve. J Physiol 117: 500-544.

86. Hladky SB, Haydon DA (1972) Ion transfer across lipid membranes in the presence of gramicidin A. I. Studies of the unit conductance channel. Biochim Biophys Acta 274: 294-312.

87. Jaffe LF (1977) Electrophoresis along cell membranes. Nature 265: 600-602.

88. Hoshino Y, Koide H, Furuya K, Haberaecker WW 3rd, Lee SH, et al. (2012) The rational design of a synthetic polymer nanoparticle that neutralizes a toxic peptide in vivo. Proc Natl Acad Sci U S A 109: 33-38

89. Sellergren B (1989) Molecular imprinting by noncovalent interactions: Tailormade chiral stationary phases of high selectivity and sample load capacity. Chirality 1: 63-68.

90. Carboni D, Malfatti L, Pinna A, Lasio B, Tokudome Y, Takahashi M, et al. (2013) Molecularly imprinted La-doped mesoporous titania films with hydrolytic properties toward organophosphate pesticides New J Chem : 1-8.

91. Hayden O, Haderspöck C, Krassnig S, Chen X, Dickert FL (2006) Surface imprinting strategies for the detection of trypsin. Analyst 131: 1044-1050.

92. LÃammerhofer M, Gargano A (2010) Monoliths with chiral surface functionalization for enantioselective capillary electrochromatography. J Pharm Biomed Anal 53: 1091-1123.

93. Dickert FL, Hayden O, Halikias KP (2001) Synthetic receptors as sensor coatings for molecules and living cells. Analyst 126: 766-771.

94. Scheuplein RJ, Blank IH (1965) Mechanism of percutaneous absorption. III. The effect of temperature on the transport of non-electrolytes across the skin. $J$ Invest Dermatol 45: 334-346.

95. Eersels K, van Grinsven B, Ethirajan A, Timmermans S, Jimenez Monroy KL, et al. (2013) Selective Identification of Macrophages and Cancer Cells Based on Thermal Transport through Surface-Imprinted Polymer Layers. ACS Appl Mater Interfaces.

96. Reddy IK, Mehvar R (2004) Chirality in drug design and development. Marce Dekker, New York, USA: 1-441.

97. Heard CM, Brain KR (1995) Does solute stereochemistry influence percutaneous penetration? Chirality $7: 305-309$.

98. Pfeiffer CC (1956) Optical isomerism and pharmacological action, a generalization. Science 124: 29-31.

99. Albery WJ, Hadgraft J (1979) Percutaneous absorption: in vivo experiments. $J$ Pharm Pharmacol 31: 140-147.

100.Sims B, Holder SL (1974) Surface activity of prostaglandins E2, F2alpha, A1, and B1 in presence of insoluble monomolecular films. J Pharm Sci 63: 15401545.

101.Pathirana S, Neely WC, Myers LJ, Vodyanoy V (1992) Chiral recognition of odorants (+)- and (-)-carvone by phospholipid monolayers. J Am Soc 114: 1404-1405.
102. Franz TJ (1975) Percutaneous absorption on the relevance of in vitro data. $J$ Invest Dermatol 64: 190-195

103. Wester RC, Maibach HI (1985) Bronaugh RL, Maiback HI (eds) In Percutaneous adsorption, Marcel Dekker: New York :251-266.

104.Gibalbi M, Perrier D (1982) Pharmacokinetics. New York: Marcel Dekker: 1-239.

105. Fromherz $P$ (1988) Self-organization of the fluid mosaic of charged channe proteins in membranes. Proc Natl Acad Sci U S A 85: 6353-6357.

106. McCracken NW, Blain PG, Williams FM (1993) Human xenobiotic metabolizing esterases in liver and blood. Biochem Pharmacol 46: 1125-1129.

107. Furtado L, Souza RLR, Tsuneto LT, Petzl-Erler ML, Chautard-Freire-Maia EA (2006) Butyrylcholinesterase genetic variability in Guarani Amerindians from the Brazilian state of Mato Grosso do Sul. Genet Mol Biol 29: 8-13.

108. Kell DB, Dobson PD, Bilsland E, Oliver SG (2013) The promiscuous binding of pharmaceutical drugs and their transporter-mediated uptake into cells: what we (need to) know and how we can do so. Drug Discov Today 18: 218-239.

109. Liu JQ, Wulff G (2004) Molecularly imprinted polymers with strong carboxypeptidase a-like activity: combination of an amidinium function with a zinc-ion binding site in transition-state imprinted cavities. Angew Chem Int Ed Engl 43: 1287-1290

110. Heard CM, Watkinson AC, Brain KR, Hadgraft J (1993) In vitro penetration of propranolol eantiomers. Int J Pharm 90: R5-R8.

111. Sellegren B, Lepisto M, Mosbach K (1988) Highly enantioselective and substrate-selective polymers obtained by molecular imprinting utilizing noncovalent interactions. NMR and chromatographic studies on the nature of recognition. J Am Chem Soc 110: 5853-5860.

112. Nakanishi K, Sakiyama T, Kumada Y, Imamura K, Imanaka H (2008) Recent advances in controlled immobilization of proteins onto the surface of the solid substrate and its possible application to proteomics. Current Proteomics 5: $161-175$.

113. Grubauer G, Feingold KR, Elias PM (1987) Relationship of epidermal lipogenesis to cutaneous barrier function. J Lipid Res 28: 746-752.

114. Feingold KR (1991) The regulation and role of epidermal lipid synthesis. Adv Lipid Res 24: 57-82.

115. Elias PM, Menon GK (1991) Structural and lipid biochemical correlates of the epidermal permeability barrier. Adv Lipid Res 24: 1-26.

116. Touitou E, Godin B, Kommuru TR, Afouna MI, Reddy IK (2004) Transport of chiral molecules across the skin. In: Chirality in drug design and development. Reddy and Mehvar (Eds), Marcel Dekker, Inc., New York: 67-99.

117. Liechty WB, Kryscio DR, Slaughter BV, Peppas NA (2010) Polymers for drug delivery systems. Annu Rev Chem Biomol Eng 1: 149-173.

118. Davies J, Davies D (2010) Origins and evolution of antibiotic resistance. Microbiol Mol Biol Rev 74: 417-433.

119. Oberdörster G, Maynard A, Donaldson K, Castranova V, Fitzpatrick J, Ausman $\mathrm{K}$, et al. (2005) Principles for characterizing the potential human health effects from exposure to nanomaterials: elements of a screening strategy. Particle Fibre Toxicol: 1-35

120.Scholes CA, Kentish SE, Stevens GW (2008) Carbon dioxide separation through polymeric membrane systems for flue gas applications. Recent Patents Chem Eng 1:52-66.

121. Lin JH, Lu AY (1997) Role of pharmacokinetics and metabolism in drug discovery and development. Pharmacol Rev 49: 403-449.

122. Nostrum CF van (2005) Molecular imprinting: A new tool for drug innovation Drug Discovery Today 2: 119-124 